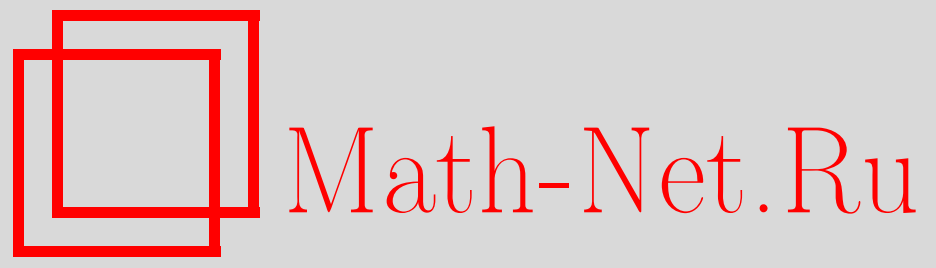

М. Е. Широков, О свойствах квантовых каналов, связанных с классической пропускной способностью, Теория вероятн. и ее примен., 2007, том 52, выпуск 2, 301335

DOI: https://doi.org/10.4213/tvp174

Использование Общероссийского математического портала Math-Net.Ru подразумевает, что вы прочитали и согласны с пользовательским соглашением

http://www.mathnet.ru/rus/agreement

Параметры загрузки:

IP : 54.237 .59 .107

26 апреля 2023 г., 14:52:25

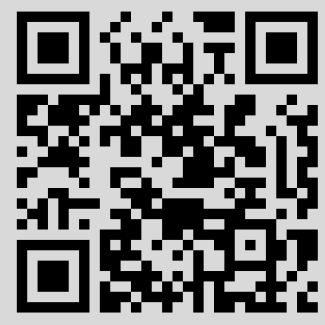




\section{О СВОЙСТВАХ КВАНТОВЫХ КАНАЛОВ, СВЯЗАННЫХ С КЛАССИЧЕСКОЙ ПРОПУСКНОЙ СПОСОБНОСТЬЮ ${ }^{1)}$}

Настояшая работа посвяшена дальнейшему изучению $\chi$-пропускной способности (the Holevo capacity) квантового канала бесконечной размерности. Показано существование и единственность выходного оптимального среднего состояния для квантового канала с ограничением, определяемым произвольным выпуклым подмножеством состояний. Получено минимаксное выражение для $\chi$-пропускной способности.

Рассматриваются $\chi$-функция и выпуклое замыкание выходной энтропии квантового канала бесконечной размерности. Показано, что $\chi$-функция произвольного канала является вогнутой полунепрерывной снизу функцией на множестве всех квантовых состояний и имеет непрерывные сужения на подмножества состояний с непрерывной выходной энтропией. Получено явное представление для выпуклого замыкания выходной энтропии и исследованы его свойства. Показано, что выпуклое замыкание выходной энтропии совпадает с выпуклой оболочкой выходной энтропии на множестве всех состояний с конечной выходной энтропией и, подобно $\chi$-функции, имеет непрерывные сужения на подмножества состояний с непрерывной выходной энтропией. Рассмотрены приложения полученных результатов в теории сцепленности. Доказанные свойства выпуклого замыкания выходной энтропии позволили обобщить на случай бесконечномерных каналов некоторые результаты, связанные с проблемой аддитивности и ранее полученные для каналов конечной размерности.

Ключевые слова и фразы: квантовое состояние, энтропия, квантовый канал, $\chi$-пропускная способность, $\chi$-функция, выпуклое замыкание выходной энтропии квантового канала.

1. Введение. Одним из основных понятий квантовой теории информации является понятие квантового канала - вполне положительного сохраняющего след отображения множества состояний входной квантовой системы в множество состояний выходной системы. Квантовые каналы характеризуются целым набором пропускных способностей,

* Математический институт им. В. А. Стеклова РАН, ул. Губкина, 8, 119991 Москва, ГСП-1, Россия; e-mail: msh@mi.ras.ru

1) Работа выполнена при поддержке научной программы Отделения математики $\mathrm{PAH} \mathrm{«Современные} \mathrm{проблемы} \mathrm{теоретической} \mathrm{математики»} \mathrm{и} \mathrm{РФФИ} \mathrm{(проект} \mathrm{№} \mathrm{06-01-}$ 00164-a). 
определяемых видом передаваемой информации (квантовой или классической), а также дополнительными ресурсами, используемыми при передаче [7], [25].

Несмотря на то, что основное внимание до последнего времени уделялось квантовым информационным системам конечной размерности, интерес к бесконечномерным квантовым системам постоянно растет. Для математически строгого исследования таких систем необходим подход, основанный на использовании общей теории меры в сепарабельных метрических пространствах, теории операторов в гильбертовом пространстве и бесконечномерного выпуклого анализа.

Настоящая работа посвящена изучению $\chi$-пропускной способности ${ }^{2)}$ квантовых каналов бесконечной размерности, а также связанных с ней энтропийных характеристик квантовых каналов и является логическим продолжением работ [9], [10], [21], [31].

$\mathrm{C}$ математической точки зрения существенным отличием квантовых каналов бесконечной размерности является некомпактность пространства квантовых состояний и отсутствие свойств конечности и непрерывности выходной энтропии. Это приводит к тому, что, даже при конечном значении $\chi$-пропускной способности, для таких каналов, вообще говоря, не существует оптимальных ансамблей, свойства которых играют существенную роль при изучении конечномерных каналов [29]. В [10] дано достаточное условие существования оптимальной меры обобщенного ансамбля для произвольного канала с ограничениями, но в [10] и [32] приведены примеры, показывающие, что оптимальная мера существует не всегда. В разделе 3 настоящей работы показано, что для произвольного канала с ограничениями, определяемыми выпуклыми подмножествами состояний, независимо от наличия оптимальной меры существует состояние, названное выходным оптимальным средним, которое обладает свойствами образа среднего состояния оптимального ансамбля для канала конечной размерности (предложение 1). Там же получено минимаксное выражение для $\chi$-пропускной способности и дано альтернативное определение выходного оптимального среднего состояния как единственной точки минимума некоторой полунепрерывной снизу функции на компактном множестве (предложение 2).

В разделе 4 вводится понятие $\chi$-функции квантового канала бесконечной размерности. При помощи выходного оптимального среднего состояния получен бесконечномерный аналог неравенства для $\chi$-функции, которое было доказано в [21] для конечномерных каналов (предложение 3 ). Показано, что $\chi$-функция произвольного канала является во-

2) Эта величина, называемая в зарубежной литературе the Holevo capacity, тесно связана с пропускной способностью для передачи классической информации по квантовому каналу связи, см., например, [7]. 
гнутой полунепрерывной снизу функцией, обладающей естественными цепными свойствами (предложения 4-5). Для $\chi$-функции получен аналог теоремы Саймона о мажорируемой сходимости (следствие 3 ).

Другая важная характеристика квантового канала - выпуклое замыкание выходной энтропии - рассмотрена в разделе 5. Поскольку в конечномерном случае выходная энтропия является непрерывной функцией на компактном множестве состояний, ее выпуклая оболочка совпадает с выпуклым замыканием [4] (нижней огибающей в терминах [12]). Важная роль этой функции при изучении конечномерных квантовых каналов обусловлена ее тесной связью с $\chi$-функцией: последняя равна разности между выходной энтропией и ее выпуклой оболочкой (замыканием). В бесконечномерном случае указанное выше совпадение, вообще говоря, не имеет места, и представляется естественным вместо выпуклой оболочки рассматривать выпуклое замыкание выходной энтропии. Получено явное представление для выпуклого замыкания выходной энтропии произвольного бесконечномерного канала и исследованы его свойства (предложения 6-8, следствие 4). Основная техническая проблема - некомпактность множества состояний - делает невозможным использование общей теории интегральных представлений на компактных выпуклых множествах [2], [12]. Главный инструмент приведенного подхода - критерий секвенциальной компактности подмножеств мер на пространстве состояний, а также связанные с ним результаты, полученные в [10]. Показано, что выпуклое замыкание выходной энтропии совпадает с ее выпуклой оболочкой на множестве состояний с конечной выходной энтропией. Таким образом, на этом множестве имеет место представление $\chi$-функции в виде разности выходной энтропии и ее выпуклого замыкания.

В разделе 6 на основе двух предыдущих разделов получен следующий результат о непрерывности: $\chi$-функция и выпуклое замыкание выходной энтропии произвольного квантового канала имеют непрерывные сужения на любое множество непрерывности выходной энтропии (теорема 1). В частности, из этого утверждения и результата работы [10] следует непрерывность $\chi$-функции для гауссовских каналов с ограничениями на среднюю энергию.

Раздел 7 посвящен проблеме аддитивности $\chi$-пропускной способности - одной из основных открытых проблем квантовой теории информации. Полученные в предыдущих разделах результаты позволили доказать бесконечномерные варианты теорем из [21] и [30] об эквивалентности различных свойств аддитивности для двух произвольных квантовых каналов.

Важный частный случай выпуклого замыкания выходной энтропии конечномерного квантового канала - это специальная мера сцепленности состояния составной системы, которую принято называть сцеп- 
ленностью формирования (Entanglement of Formation, EoF) [17]. В конечномерном случае EoF совпадает с выпуклым замыканием выходной энтропии частичного следа - канала из пространства состояний составной системы в пространство состояний подсистемы. В разделе 8 рассматриваются аргументы в пользу определения EoF для состояния в тензорном произведении двух квантовых систем бесконечной размерности как выпуклого замыкания выходной энтропии частичного следа. Показано, что такое определение является естественным и гарантирует такие свойства EoF, как выпуклость, полунепрерывность снизу на всем пространстве состояний, а также непрерывность на множестве состояний с ограниченной средней энергией. Показано, что это определение совпадает с принятым в [19] для всех состояний с конечной выходной энтропией частичных состояний. Вопрос об их совпадении на множестве всех состояний остается пока открытым.

2. Предварительные сведения. Пусть $\mathscr{H}$ - сепарабельное гильбертово пространство, $\mathfrak{B}(\mathscr{H})$ - алгебра всех ограниченных операторов в $\mathscr{H}, \mathfrak{T}(\mathscr{H})$ - банахово пространство всех ядерных операторов со следовой нормой $\|\cdot\|_{1}$. Состоянием далее называется положительный ядерный оператор $\rho$ в $\mathscr{H}$ с единичным следом: $\rho \geqslant 0 ; \operatorname{Tr} \rho=1$. Алгебра $\mathfrak{B}(\mathscr{H})$ обычно называется алгеброй наблюдаемых квантовой системы, тогда состояние $\rho$ задает функционал математического ожидания $A \mapsto \operatorname{Tr} \rho A ; A \in \mathfrak{B}(\mathscr{H})$, т.е. нормальное состояние в смысле теории операторных алгебр [2]. Множество всех состояний $\mathfrak{S}(\mathscr{H})$ - выпуклое замкнутое подмножество $\mathfrak{T}(\mathscr{H})$, которое является полным сепарабельным метрическим пространством с метрикой, определяемой следовой нормой.

Конечный набор состояний $\left\{\rho_{i}\right\}$ с соответствующими вероятностями $\left\{\pi_{i}\right\}$ называется (конечным) ансамблем и обозначается $\left\{\pi_{i}, \rho_{i}\right\}$, а состояние $\bar{\rho}=\sum_{i} \pi_{i} \rho_{i}$ называется средним состоянием этого ансамбля. В [10] введено понятие обобщенного ансамбля как произвольной вероятностной борелевской меры $\pi$ на $\mathfrak{S}(\mathscr{H})$. Средним состоянием обобщенного ансамбля $\pi$ называется состояние (это состояние называется также барицентром меры $\pi$ ), определяемое интегралом Бохнера

$$
\bar{\rho}(\pi)=\int_{\mathfrak{S}(\mathscr{H})} \rho \pi(d \rho) .
$$

Обычное понятие ансамбля соответствует мерам с конечным носителем.

Под выпуклой комбинацией ансамблей будем понимать выпуклую комбинацию соответствующих этим ансамблям мер. В частности, для любого набора ансамблей $\left\{\left\{\pi_{i}^{k}, \rho_{i}^{k}\right\}_{i=1}^{n(k)}\right\}_{k=1}^{m}$ и любого распределения вероятностей $\left\{\lambda_{k}\right\}_{k=1}^{m}$ ансамбль, состоящий из $\sum_{k=1}^{m} n(k)$ состояний $\left\{\rho_{i}^{k}\right\}_{k, i}$ с соответствующими вероятностями $\left\{\lambda_{k} \pi_{i}^{k}\right\}_{k, i}$ является выпуклой комбинацией этих ансамблей и обозначается $\sum_{k=1}^{m} \lambda_{k}\left\{\pi_{i}^{k}, \rho_{i}^{k}\right\}_{i=1}^{n(k)}$. 
Пусть $\mathscr{P}$ - выпуклое множество всех вероятностных мер на $\mathfrak{S}(\mathscr{H})$, снабженное топологией слабой сходимости [1]. Как отмечено в [10], отображение $\mathscr{P} \ni \pi \mapsto \bar{\rho}(\pi)$ непрерывно в этой топологии. Подмножество $\mathscr{P}$, состоящее из всех мер $\pi$ с барицентром $\bar{\rho}(\pi)$ в $\mathscr{A} \subseteq \mathfrak{S}(\mathscr{H})$, обозначим $\mathscr{P}_{\mathscr{A}}$.

Пусть $A$ и $B$ - положительные операторы из $\mathfrak{T}(\mathscr{H})$. Энтропия фон Неймана оператора $A$ и относительная энтропия операторов $A$ и $B$ определяются соответственно выражениями

$H(A)=-\sum_{i}\langle i|A \log A| i\rangle \quad$ и $\quad H(A \| B)=\sum_{i}\langle i|A \log A-A \log B+B-A| i\rangle$,

в которых $\{|i\rangle\}$ - базис из собственных векторов оператора $A$ (подробнее см. в [22], [34]). Энтропия и относительная энтропия являются полунепрерывными снизу функциями своих аргументов со значениями в $[0 ;+\infty]$, первая из которых вогнута, а вторая выпукла по совокупности аргументов [22], [34]. Отметим также следующее неравенство:

$$
H(\rho \| \sigma) \geqslant \frac{1}{2}\|\rho-\sigma\|_{1}^{2},
$$

которое имеет место для произвольных состояний $\rho$ и $\sigma$ из $\mathfrak{S}(\mathscr{H})$ [27].

Относительную энтропию $H(\rho \| \sigma)$ двух состояний $\rho$ и $\sigma$ можно рассматривать как меру различия этих состояний, классический аналог которой называется расстоянием Кульбака-Лейблера. Несмотря на то, что эта мера не является метрикой (она не симметрична и не удовлетворяет аксиоме треугольника), можно ввести понятие сходимости последовательности состояний $\left\{\rho_{n}\right\}$ к некоторому состоянию $\rho_{*}$, определяемое условием $\lim _{n \rightarrow+\infty} H\left(\rho_{n} \| \rho_{*}\right)=0$. Топология на пространстве состояний, связанная с такой сходимостью, в классическом случае подробно изучалась в [20], где была названа сильной информационной топологией. В квантовой теории информации этот вид сходимости, который для краткости будем называть $H$-сходимостью, также играет важную роль (см. $[11$, предложение 2]). Из неравенства (1) следует, что

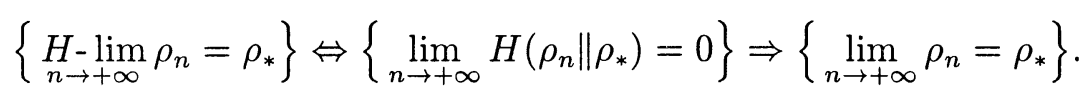

В дальнейшем неоднократно будет использоваться тождество Дональда [15], [27]

$$
\sum_{i=1}^{n} \pi_{i} H\left(\rho_{i} \| \widehat{\rho}\right)=\sum_{i=1}^{n} \pi_{i} H\left(\rho_{i} \| \bar{\rho}\right)+H(\bar{\rho} \| \widehat{\rho}),
$$

которое имеет место для произвольного ансамбля $\left\{\pi_{i}, \rho_{i}\right\}_{i=1}^{n}$ со средним $\bar{\rho}$ и произвольного состояния $\widehat{\rho}$.

Пусть $\mathscr{H}, \mathscr{H}^{\prime}$ - пара сепарабельных гильбертовых пространств, которые мы будем называть входным и выходным пространствами соответственно. Канал $\Phi$ - это линейное положительное сохраняющее 
след отображение $\mathfrak{T}(\mathscr{H})$ в $\mathfrak{T}\left(\mathscr{H}^{\prime}\right)$ такое, что двойственное отображение $\Phi^{*}: \mathfrak{B}\left(\mathscr{H}^{\prime}\right) \mapsto \mathfrak{B}(\mathscr{H})$ (которое существует, поскольку $\Phi$ ограничено) является вполне положительным, см. [8, гл. 3, п. 1]. В частности, канал отображает входные состояния из $\mathfrak{S}(\mathscr{H})$ в выходные состояния из $\mathfrak{S}\left(\mathscr{H}^{\prime}\right)$.

Важной характеристикой канала $\Phi$ является его выходная энтропия $H_{\Phi}(\rho)=H(\Phi(\rho))$ - вогнутая полунепрерывная снизу неотрицательная функция на множестве входных состояний $\mathfrak{S}(\mathscr{H})$.

Пусть $\mathscr{A}$ - произвольное подмножество $\mathfrak{S}(\mathscr{H})$. Рассмотрим ограничение на входной ансамбль $\left\{\pi_{i}, \rho_{i}\right\}$, определяемое включением $\bar{\rho} \in \mathscr{A}$. Канал $\Phi$ с таким ограничением называется $\mathscr{A}$-ограниченнылм каналом. $\chi$-пропускная способность $\mathscr{A}$-ограниченного канала $\Phi$ определяется следующим образом [9], [10]:

$$
\bar{C}(\Phi, \mathscr{A})=\sup _{\bar{\rho} \in \mathscr{A}} \chi_{\Phi}\left(\left\{\pi_{i}, \rho_{i}\right\}\right)
$$

где

$$
\chi_{\Phi}\left(\left\{\pi_{i}, \rho_{i}\right\}\right)=\sum_{i} \pi_{i} H\left(\Phi\left(\rho_{i}\right) \| \Phi(\bar{\rho})\right)
$$

В [10] показано, что $\chi$-пропускную способность $\mathscr{A}$-ограниченного канала $\Phi$ можно также определить выражением

$$
\bar{C}(\Phi, \mathscr{A})=\sup _{\pi \in \mathscr{P}_{\mathscr{A}}} \int_{\mathfrak{S}(\mathscr{H})} H(\Phi(\rho) \| \Phi(\bar{\rho}(\pi))) \pi(d \rho),
$$

что означает совпадение точной верхней грани по всем мерам из $\mathscr{P} \mathscr{A}$ с точной верхней гранью по всем мерам из $\mathscr{P}_{\mathscr{A}}$ с конечным носителем.

3. Оптимальное среднее. Как известно, для произвольного конечномерного канала $\Phi$ и произвольного замкнутого множества $\mathscr{A}$ существует оптимальный ансамбль $\left\{\pi_{i}, \rho_{i}\right\}$, на котором достигается точная верхняя грань в определении (3) $\chi$-пропускной способности [18], [29]. Образ среднего состояния оптимального ансамбля играет важную роль при исследовании конечномерных каналов [21].

Для квантовых каналов бесконечной размерности обычно не существует оптимальных конечных ансамблей. Поэтому естественно ввести понятие обобщенного оптимального ансамбля - оптимальной меры на множестве всех состояний, на которой достигается точная верхняя грань в определении (5) $\chi$-пропускной способности. В [10] дано достаточное условие существования оптимальной меры для произвольного канала с ограничениями, но примеры в [10], [32] показывают, что оптимальная мера существует не всегда.

Цель данного раздела - независимо от наличия оптимальной меры показать существование и единственность такого состояния, которое 
обладает всеми основными свойствами образа среднего состояния оптимального ансамбля для конечномерного канала. Такое состояние естественно назвать выходным оптимальным средним для канала с ограничением. Если оптимальная мера существует, то образ ее среднего состояния - барицентра является выходным оптимальным средним в указанном выше смысле. Используя свойства этого состояния, можно обобщить некоторые результаты [21] на случай бесконечномерных каналов.

Лемма 1. Пусть $\left\{\left\{\pi_{i}^{k}, \rho_{i}^{k}\right\}_{i=1}^{n(k)}\right\}_{k=1}^{m}$ - конечныцй набор ансамблей, $a\left\{\lambda_{k}\right\}_{k=1}^{m}$ - распределение вероятностей. Тогда

$$
\chi_{\Phi}\left(\sum_{k=1}^{m} \lambda_{k}\left\{\pi_{i}^{k}, \rho_{i}^{k}\right\}_{i=1}^{n(k)}\right)=\sum_{k=1}^{m} \lambda_{k} \chi_{\Phi}\left(\left\{\pi_{i}^{k}, \rho_{i}^{k}\right\}_{i=1}^{n(k)}\right)+\chi_{\Phi}\left(\left\{\lambda_{k}, \bar{\rho}_{k}\right\}_{k=1}^{m}\right),
$$

где $\bar{\rho}_{k}$ - среднее состояние ансамбля $\left\{\pi_{i}^{k}, \rho_{i}^{k}\right\}_{i=1}^{n(k)}, k=1, \ldots, m$.

При $m=2$ для любого $\lambda \in[0,1]$ имеет место неравенство

$$
\begin{aligned}
\chi_{\Phi}( & \left.\lambda\left\{\pi_{i}^{1}, \rho_{i}^{1}\right\}_{i=1}^{n(1)}+(1-\lambda)\left\{\pi_{i}^{2}, \rho_{i}^{2}\right\}_{i=1}^{n(2)}\right) \geqslant \lambda \chi_{\Phi}\left(\left\{\pi_{i}^{1}, \rho_{i}^{1}\right\}_{i=1}^{n(1)}\right) \\
& +(1-\lambda) \chi_{\Phi}\left(\left\{\pi_{i}^{2}, \rho_{i}^{2}\right\}_{i=1}^{n(2)}\right)+\frac{\lambda(1-\lambda)}{2}\left\|\Phi\left(\bar{\rho}_{2}\right)-\Phi\left(\bar{\rho}_{1}\right)\right\|_{1}^{2} .
\end{aligned}
$$

Д о к а з а т е л ь с т в о. Пусть $\bar{\rho}=\sum_{k=1}^{m} \lambda_{k} \bar{\rho}_{k}$ - среднее состоянйе ансамбля $\sum_{k=1}^{m} \lambda_{k}\left\{\pi_{i}^{k}, \rho_{i}^{k}\right\}_{i=1}^{n(k)}$. По определению

$$
\chi_{\Phi}\left(\sum_{k=1}^{m} \lambda_{k}\left\{\pi_{i}^{k}, \rho_{i}^{k}\right\}_{i=1}^{n(k)}\right)=\sum_{k=1}^{m} \lambda_{k} \sum_{i=1}^{n(k)} \pi_{i}^{k} H\left(\Phi\left(\rho_{i}^{k}\right) \| \Phi(\bar{\rho})\right) .
$$

Применяя тождество Дональда (2) к каждой внутренней сумме в правой части данного выражения, получаем основное тождество леммы.

Для доказательства неравенства при $m=2$ достаточно применить неравенство (1) для оценки снизу последнего слагаемого в правой части основного тождества леммы:

$$
\begin{aligned}
\lambda & H\left(\Phi\left(\bar{\rho}_{1}\right) \| \Phi\left(\lambda \bar{\rho}_{1}+(1-\lambda) \bar{\rho}_{2}\right)\right)+(1-\lambda) H\left(\Phi\left(\bar{\rho}_{2}\right) \| \Phi\left(\lambda \bar{\rho}_{1}+(1-\lambda) \bar{\rho}_{2}\right)\right) \\
& \geqslant \frac{1}{2} \lambda\left\|(1-\lambda) \Phi\left(\bar{\rho}_{2}-\bar{\rho}_{1}\right)\right\|_{1}^{2}+\frac{1}{2}(1-\lambda)\left\|\lambda \Phi\left(\bar{\rho}_{2}-\bar{\rho}_{1}\right)\right\|_{1}^{2} \\
& =\frac{1}{2} \lambda(1-\lambda)\left\|\Phi\left(\bar{\rho}_{2}\right)-\Phi\left(\bar{\rho}_{1}\right)\right\|_{1}^{2} .
\end{aligned}
$$

Лемма 1 доказана.

Несмотря на возможное отсутствие оптимального ансамбля для $\mathscr{A}$-ограниченного канала $\Phi$, из определения $\chi$-пропускной способности следует существование последовательности ансамблей с указанными ниже свойствами.

О п р е д е л е н и е 1. Последовательность ансамблей $\left\{\left\{\pi_{i}^{k}, \rho_{i}^{k}\right\}\right\}_{k}$ такая, что $\bar{\rho}_{k}=\sum_{i} \pi_{i}^{k} \rho_{i}^{k} \in \mathscr{A}$ для всех $k$ и $\lim _{k \rightarrow+\infty} \chi_{\Phi}\left(\left\{\pi_{i}^{k}, \rho_{i}^{k}\right\}\right)=$ 
$\bar{C}(\Phi, \mathscr{A})$, называется аппроксимирующей последовательностью для $\mathscr{A}$-ограниченного канала $\Phi$.

Состояние называется входньл оптимальным средним для $\mathscr{A}$-ограниченного канала $\Phi$, если оно является пределом последовательности средних состояний некоторой аппроксимирующей последовательности ансамблей для $\mathscr{A}$-ограниченного канала $\Phi$.

Данное определение не гарантирует ни существования, ни единственности входного оптимального среднего (пример канала, у которого не существует входных оптимальных средних, рассмотрен в [3]). Наличие по крайней мере одного входного оптимального среднего является необходимым, а при дополнительных условиях непрерывности выходной энтропии и достаточным условием существования оптимальной меры для $\mathscr{A}$-ограниченного канала $\Phi$, причем это входное оптимальное среднее является барицентром оптимальной меры (см. подробности в [10]).

Несмотря на возможное отсутствие предельных точек у последовательности средних состояний аппроксимирующей последовательности ансамблей для $\mathscr{A}$-ограниченного канала, конечность его $\chi$-пропускной способности гарантирует сходимость последовательности образов этих средних.

Следующее предложение является обобщением предложения 1 из [31] на случай некомпактного множества $\mathscr{A}$.

Предложение 1. Пусть $\Phi: \mathfrak{S}(\mathscr{H}) \mapsto \mathfrak{S}\left(\mathscr{H}^{\prime}\right)-$ произвольный канал, а $\mathscr{A}$ - выпуклое подмножество множества $\mathfrak{S}(\mathscr{H})$ такое, что $\bar{C}(\Phi, \mathscr{A})<+\infty$. Тогда существует единственное состояние $\Omega(\Phi, \mathscr{A})$ в $\mathfrak{S}\left(\mathscr{H}^{\prime}\right)$ mакое, что $\sum_{j} \mu_{j} H\left(\Phi\left(\sigma_{j}\right) \| \Omega(\Phi, \mathscr{A})\right) \leqslant \bar{C}(\Phi, \mathscr{A})$ для любого ансамбля $\left\{\mu_{j}, \sigma_{j}\right\}$ со средним состоянием $\bar{\sigma} \in \mathscr{A}$.

Состояние $\Omega(\Phi, \mathscr{A})$ лежит в $\overline{\Phi(\mathscr{A})}$. Для любой аппроксимирующей последовательности ансамблей $\left\{\left\{\pi_{i}^{k}, \rho_{i}^{k}\right\}\right\}_{k}$ для $\mathscr{A}$-ограниченного канала $\Phi$ с соответствующей последовательностью средних состояний $\left\{\bar{\rho}_{k}\right\}_{k}$ существует

$$
\underset{k \rightarrow+\infty}{H-\lim _{k}} \Phi\left(\bar{\rho}_{k}\right)=\Omega(\Phi, \mathscr{A}) \text {. }
$$

Д о к а з а т е л с т в П. Покажем прежде всего, что для любой аппроксимирующей последовательности ансамблей $\left\{\Sigma_{k}=\left\{\pi_{i}^{k}, \rho_{i}^{k}\right\}_{i=1}^{n(k)}\right\}$ для $\mathscr{A}$-ограниченного канала $\Phi$ последовательность $\left\{\Phi\left(\bar{\rho}_{k}\right)\right\}$ сходится к некоторому состоянию из $\mathfrak{S}\left(\mathscr{H}^{\prime}\right)$. По определению аппроксимирующей последовательности для любого $\varepsilon>0$ существует $N_{\varepsilon}$ такое, что $\chi_{\Phi}\left(\Sigma_{k}\right)>\bar{C}(\Phi, \mathscr{A})-\varepsilon$ для любого $k \geqslant N_{\varepsilon}$. В силу леммы 1 (при $m=2$ и $\lambda=\frac{1}{2}$ ) для любых $k_{1} \geqslant N_{\varepsilon}$ и $k_{2} \geqslant N_{\varepsilon}$ имеем

$$
\begin{aligned}
\bar{C}(\Phi, \mathscr{A})-\varepsilon \leqslant & \frac{1}{2} \chi_{\Phi}\left(\Sigma_{k_{1}}\right)+\frac{1}{2} \chi_{\Phi}\left(\Sigma_{k_{2}}\right) \leqslant \chi_{\Phi}\left(\frac{1}{2} \Sigma_{k_{1}}+\frac{1}{2} \Sigma_{k_{2}}\right) \\
& -\frac{1}{8}\left\|\Phi\left(\bar{\rho}_{k_{2}}\right)-\Phi\left(\bar{\rho}_{k_{1}}\right)\right\|_{1}^{2} \leqslant \bar{C}(\Phi, \mathscr{A})-\frac{1}{8}\left\|\Phi\left(\bar{\rho}_{k_{2}}\right)-\Phi\left(\bar{\rho}_{k_{1}}\right)\right\|_{1}^{2},
\end{aligned}
$$


и, следовательно, $\left\|\Phi\left(\bar{\rho}_{k_{2}}\right)-\Phi\left(\bar{\rho}_{k_{1}}\right)\right\|_{1}<\sqrt{8 \varepsilon}$. Таким образом, последовательность $\left\{\Phi\left(\bar{\rho}_{k}\right)\right\}$ фундаментальна, а значит, сходится к некоторому состоянию $\omega$ из $\mathfrak{S}\left(\mathscr{H}^{\prime}\right)$.

Пусть $\left\{\mu_{j}, \sigma_{j}\right\}_{j=1}^{m}$ - произвольный ансамбль из $m$ состояний со средним $\bar{\sigma} \in \mathscr{A}$. Рассмотрим семейство ансамблей

$$
\Sigma_{k}^{\lambda}=(1-\lambda)\left\{\pi_{i}^{k}, \rho_{i}^{k}\right\}_{i=1}^{n(k)}+\lambda\left\{\mu_{j}, \sigma_{j}\right\}_{j=1}^{m}, \quad \lambda \in[0,1], \quad k \in \mathbf{N},
$$

со средними состояниями $\bar{\rho}_{k}^{\lambda}=(1-\lambda) \bar{\rho}_{k}+\lambda \bar{\sigma}$. В силу выпуклости множества $\mathscr{A}$ имеем $\bar{\rho}_{k}^{\lambda} \in \mathscr{A}$ для всех $\lambda \in[0,1]$ и $k \in \mathbf{N}$, причем по доказанному выше

$$
\lim _{k \rightarrow+\infty} \Phi\left(\bar{\rho}_{k}^{\lambda}\right)=(1-\lambda) \omega+\lambda \Phi(\bar{\sigma})
$$

По определению

$$
\chi_{\Phi}\left(\Sigma_{k}^{\lambda}\right)=(1-\lambda) \sum_{i=1}^{n(k)} \pi_{i}^{k} H\left(\Phi\left(\rho_{i}^{k}\right) \| \Phi\left(\bar{\rho}_{k}^{\lambda}\right)\right)+\lambda \sum_{j=1}^{m} \mu_{j} H\left(\Phi\left(\sigma_{j}\right) \| \Phi\left(\bar{\rho}_{k}^{\lambda}\right)\right) .
$$

В силу условия $\bar{C}(\Phi, \mathscr{A})<+\infty$ обе суммы в правой части данного выражения конечны. Применяя тождество Дональда (2) к первой сумме в правой части, получаем

$$
\sum_{i=1}^{n(k)} \pi_{i}^{k} H\left(\Phi\left(\rho_{i}^{k}\right) \| \Phi\left(\bar{\rho}_{k}^{\lambda}\right)\right)=\chi_{\Phi}\left(\Sigma_{k}^{0}\right)+H\left(\Phi\left(\bar{\rho}_{k}\right) \| \Phi\left(\bar{\rho}_{k}^{\lambda}\right)\right) .
$$

Подстановка этого выражения в (7) дает

$$
\begin{aligned}
\chi_{\Phi}\left(\Sigma_{k}^{\lambda}\right)= & \chi_{\Phi}\left(\Sigma_{k}^{0}\right)+(1-\lambda) H\left(\Phi\left(\bar{\rho}_{k}\right) \| \Phi\left(\bar{\rho}_{k}^{\lambda}\right)\right) \\
& +\lambda\left(\sum_{j=1}^{m} \mu_{j} H\left(\Phi\left(\sigma_{j}\right) \| \Phi\left(\bar{\rho}_{k}^{\lambda}\right)\right)-\chi_{\Phi}\left(\Sigma_{k}^{0}\right)\right) .
\end{aligned}
$$

Отсюда, в силу неотрицательности относительной энтропии, получаем

$$
\sum_{j=1}^{m} \mu_{j} H\left(\Phi\left(\sigma_{j}\right) \| \Phi\left(\bar{\rho}_{k}^{\lambda}\right)\right) \leqslant \lambda^{-1}\left(\chi_{\Phi}\left(\Sigma_{k}^{\lambda}\right)-\chi_{\Phi}\left(\Sigma_{k}^{0}\right)\right)+\chi_{\Phi}\left(\Sigma_{k}^{0}\right)
$$

при $\lambda \neq 0$. Из определения аппроксимирующей последовательности следует, что

$$
\lim _{k \rightarrow+\infty} \chi_{\Phi}\left(\Sigma_{k}^{0}\right)=\bar{C}(\Phi, \mathscr{A}) \geqslant \chi_{\Phi}\left(\Sigma_{k}^{\lambda}\right)
$$

для всех $k$ и всех $\lambda \in[0,1]$. Поэтому

$$
\liminf _{\lambda \rightarrow+0} \liminf _{k \rightarrow+\infty} \lambda^{-1}\left(\chi_{\Phi}\left(\Sigma_{k}^{\lambda}\right)-\chi_{\Phi}\left(\Sigma_{k}^{0}\right)\right) \leqslant 0 .
$$

В силу полунепрерывности снизу относительной энтропии, из (6), (8), (9) и (10) следует, что

$$
\sum_{j=1}^{m} \mu_{j} H\left(\Phi\left(\sigma_{j}\right) \| \omega\right) \leqslant \liminf _{\lambda \rightarrow+0} \liminf _{k \rightarrow+\infty} \sum_{j=1}^{m} \mu_{j} H\left(\Phi\left(\sigma_{j}\right) \| \Phi\left(\bar{\rho}_{k}^{\lambda}\right)\right) \leqslant \bar{C}(\Phi, \mathscr{A}) .
$$


Таким образом доказано, что

$$
\sum_{j} \mu_{j} H\left(\Phi\left(\sigma_{j}\right) \| \omega\right) \leqslant \bar{C}(\Phi, \mathscr{A})
$$

для любого ансамбля $\left\{\mu_{j}, \sigma_{j}\right\}$ со средним состоянием $\bar{\sigma} \in \mathscr{A}$.

Пусть $\left\{\left\{\mu_{i}^{k}, \sigma_{i}^{k}\right\}\right\}_{k}$ - произвольная аппроксимирующая последовательность ансамблей для $\mathscr{A}$-ограниченного канала $\Phi$ с соответствующей последовательностью средних состояний $\left\{\bar{\sigma}_{k}\right\}_{k}$. Из свойства (11) следует, что

$$
\sum_{i} \mu_{i}^{k} H\left(\Phi\left(\sigma_{i}^{k}\right) \| \omega\right) \leqslant \bar{C}(\Phi, \mathscr{A}) \quad \forall k .
$$

Применяя тождество (2), получаем

$$
\sum_{i} \mu_{i}^{k} H\left(\Phi\left(\sigma_{i}^{k}\right) \| \omega\right)=\sum_{i} \mu_{i}^{k} H\left(\Phi\left(\sigma_{i}^{k}\right) \| \Phi\left(\bar{\sigma}_{k}\right)\right)+H\left(\Phi\left(\bar{\sigma}_{k}\right) \| \omega\right) .
$$

Из двух предыдущих выражений следует, что

$$
H\left(\Phi\left(\bar{\sigma}_{k}\right) \| \omega\right) \leqslant \bar{C}(\Phi, \mathscr{A})-\sum_{i} \mu_{i}^{k} H\left(\Phi\left(\sigma_{i}^{k}\right) \| \Phi\left(\bar{\sigma}_{k}\right)\right) .
$$

Правая часть данного неравенства стремится к нулю при $k \rightarrow+\infty$ в силу аппроксимирующего свойства последовательности $\left\{\left\{\mu_{i}^{k}, \sigma_{i}^{k}\right\}\right\}_{k}$, а значит, сушествует $\lim _{k \rightarrow+\infty} H\left(\Phi\left(\bar{\sigma}_{k}\right) \| \omega\right)=0$, что гарантирует сходимость последовательности $\left\{\bar{\sigma}_{k}\right\}$ к состоянию $\omega$. Таким образом, состояние $\omega$ не зависит от выбора аппроксимирующей последовательности, а определяется только каналом $\Phi$ и множеством-ограничением $\mathscr{A}$. Обозначим это состояние $\Omega(\Phi, \mathscr{A})$. Из приведенного выше рассуждения также следует, что $\omega=\Omega(\Phi, \mathscr{A})$ - единственное состояние, для которого имеет место свойство (11). Предложение 1 доказано.

Предложение 1 показывает, в частности, что множество входных оптимальных средних для $\mathscr{A}$-ограниченного канала $\Phi$ либо пусто, либо отображается каналом $\Phi$ в одно состояние.

Следствие 1. Пусть $\mathscr{A}$ - выпуклое подмножество множества $\mathfrak{S}(\mathscr{H})$. Если для $\mathscr{A}$-ограниченного канала $\Phi$ существует входное оптимальное среднее $\rho_{*}$, то $\Phi\left(\rho_{*}\right)=\Omega(\Phi, \mathscr{A})$.

Заметим, что достаточным условием существования входного оптимального среднего является компактность множества $\mathscr{A}$.

Данное следствие оправдывает следующее определение.

О п р е д е л е н и е 2. Состояние $\Omega(\Phi, \mathscr{A})$ называется выходным оптимальньм средним для $\mathscr{A}$-ограниченного канала $\Phi$.

Отметим, что существуют примеры $\mathscr{A}$-ограниченных каналов $\Phi$ с конечной $\chi$-пропускной способностью, для которых не существует входных оптимальных средних, в то время как выходное оптимальное среднее $\Omega(\Phi, \mathscr{A})$ определяется явно и играет важную роль при изучении этих каналов (см. примеры в [3], [32]). 
Существует другой подход к определению состояния $\Omega(\Phi, \mathscr{A})$. В [11] показано (следствие 6), что необходимым условием конечности $\chi$-пропускной способности $\mathscr{A}$-ограниченного канала $\Phi$ является компактность множества $\overline{\Phi(\mathscr{A})}$. Для любого ансамбля $\left\{\mu_{j}, \sigma_{j}\right\}$ со средним $\bar{\sigma} \in \mathscr{A}$ рассмотрим полунепрерывную снизу функцию $F_{\left\{\mu_{j}, \sigma_{j}\right\}}(\omega)=\sum_{j} \mu_{j} H\left(\Phi\left(\sigma_{j}\right) \| \omega\right)$ на множестве $\overline{\Phi(\mathscr{A})}$. Функция $F(\omega)=\sup _{\sum_{j} \mu_{j} \sigma_{j} \in \mathscr{A}} F_{\left\{\mu_{j}, \sigma_{j}\right\}}(\omega)$ также является полунепрерывной снизу и, следовательно, достигает своего минимума на компактном множестве $\overline{\Phi(\mathscr{A})}$. Следующее предложение показывает, в частности, что состояние $\Omega(\Phi, \mathscr{A})$ можно определить как единственную точку минимума функции $F(\omega)$ на множестве $\overline{\Phi(\mathscr{A})}$.

Предложение 2. Пусть $\Phi: \mathfrak{S}(\mathscr{H}) \mapsto \mathfrak{S}\left(\mathscr{H}^{\prime}\right)-$ произвольный канал, а $\mathscr{A}$ - выпуклое подмножество множества $\mathfrak{S}(\mathscr{H}) . \chi$-пропускная способность $\mathscr{A}$-ограниченного канала $\Phi$ определяется выражением

$$
\bar{C}(\Phi, \mathscr{A})=\inf _{\omega \in \overline{\Phi(\mathscr{A})}}\left(\sup _{\sum_{j} \mu_{j} \sigma_{j} \in \mathscr{A}} \sum_{j} \mu_{j} H\left(\Phi\left(\sigma_{j}\right) \| \omega\right)\right) .
$$

Eсли $\bar{C}(\Phi, \mathscr{A})<+\infty$, то $\Omega(\Phi, \mathscr{A})$ - единственное состояние, на котором достигается точная нижняя грань в правой части данного выражения.

Д ок аз а т е л ь с т в о. Пусть $\bar{C}(\Phi, \mathscr{A})<+\infty$. Покажем прежде всего, что

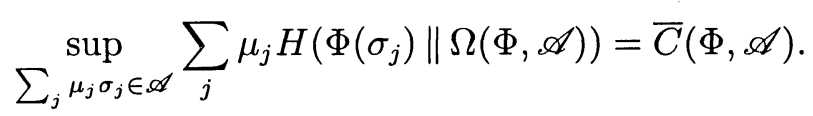

Из предложения 1 следует неравенство «ミ» в (13). Для доказательства обратного неравенства рассмотрим произвольную аппроксимирующую последовательность $\left\{\left\{\pi_{i}^{k}, \rho_{i}^{k}\right\}\right\}_{k}$. Используя тождество Дональда (2), получаем

$$
\sum_{i} \pi_{i}^{k} H\left(\Phi\left(\rho_{i}^{k}\right) \| \Omega(\Phi, \mathscr{A})\right)=\sum_{i} \pi_{i}^{k} H\left(\Phi\left(\rho_{i}^{k}\right) \| \Phi\left(\bar{\rho}_{k}\right)\right)+H\left(\Phi\left(\bar{\rho}_{k}\right) \| \Omega(\Phi, \mathscr{A})\right)
$$

для всех $k$. В силу аппроксимирующего свойства последовательности $\left\{\left\{\pi_{i}^{k}, \rho_{i}^{k}\right\}\right\}_{k}$, первое слагаемое в правой части стремится к $\bar{C}(\Phi, \mathscr{A})$ при $k \rightarrow+\infty$, а второе неотрицательно. Это доказывает неравенство «》» и, следовательно, равенство в (13).

Пусть $\omega_{*}$ - точка минимума функции $F(\omega)$. В силу равенства (13) имеем

$$
\sum_{\sum_{j}} \sup _{\mu_{j} \sigma_{j} \in \mathscr{A}} \sum_{j} \mu_{j} H\left(\Phi\left(\sigma_{j}\right) \| \omega_{*}\right)=F\left(\omega_{*}\right) \leqslant F(\Omega(\Phi, \mathscr{A}))=\bar{C}(\Phi, \mathscr{A}) .
$$

Из предложения 1 следует, что $\omega_{*}=\Omega(\Phi, \mathscr{A})$. 
Если $\bar{C}(\Phi, \mathscr{A})=+\infty$, то и правая часть выражения в предложении 2 равна $+\infty$. Действительно, если существует состояние $\omega$ из $\mathfrak{S}\left(\mathscr{H}^{\prime}\right)$ такое, что $\sup _{\sum_{j} \mu_{j} \sigma_{j} \in \mathscr{A}} \sum_{j} \mu_{j} H\left(\Phi\left(\sigma_{j}\right) \| \omega\right)<+\infty$, то из равенства (12), которое имеет место для произвольной аппроксимирующей последовательности ансамблей $\left\{\left\{\mu_{i}^{k}, \sigma_{i}^{k}\right\}\right\}_{k}$, следует $\bar{C}(\Phi, \mathscr{A})<+\infty$. Предложение 2 доказано.

3 а м е ч а н и е 1. Предположение о выпуклости множества $\mathscr{A}$ в предложениях 1, 2 и следствии 1 существенно. Рассмотрим тождественный канал $\Phi=\operatorname{Id}$ и множество $\mathscr{A}$, состоящее из двух состояний $\rho_{1}$ и $\rho_{2}$ таких, что $H\left(\rho_{1}\right)=H\left(\rho_{2}\right)<+\infty$. В этом случае $\bar{C}(\Phi, \mathscr{A})=H\left(\rho_{1}\right)=$ $H\left(\rho_{2}\right)$, состояния $\rho_{1}$ и $\rho_{2}$ являются входными оптимальными средними в смысле определения 1 с различными образами $\Phi\left(\rho_{1}\right)=\rho_{1}$ и $\Phi\left(\rho_{2}\right)=\rho_{2}$.

4. $\chi$-функция. Пусть $\Phi: \mathfrak{S}(\mathscr{H}) \mapsto \mathfrak{S}\left(\mathscr{H}^{\prime}\right)$ - произвольный канал. Рассмотрим функцию $\chi_{\Phi}$ на $\mathfrak{S}(\mathscr{H})$, которая в состоянии $\rho$ принимает значение $\bar{C}(\Phi,\{\rho\})$. Учитывая определения $\chi$-пропускной способности (3) и (5), получаем

$$
\chi_{\Phi}(\rho)=\sup _{\sum_{i} \pi_{i} \rho_{i}=\rho} \sum_{i} \pi_{i} H\left(\Phi\left(\rho_{i}\right) \| \Phi(\rho)\right)=\sup _{\pi \in \mathscr{P}_{\{\rho\}}} \int_{\mathfrak{S}(\mathscr{H})} H(\Phi(\sigma) \| \Phi(\rho)) \pi(d \sigma) .
$$

В конечномерном случае $\chi_{\Phi}$ - это непрерывная вогнутая неотрицательная функция на множестве всех состояний $\mathfrak{S}(\mathscr{H})$, которая является эффективным инструментом исследования классической пропускной способности квантового канала, в частности, проблемы аддитивности [21]. В данном разделе рассмотрены свойства $\chi$-функции произвольного бесконечномерного канала $\Phi$.

В [10] показано, что при условии $H_{\Phi}(\rho)<+\infty$ точная верхняя грань в последнем выражении в (14) достигается на некоторой мере с носителем на множестве чистых состояний.

О п р е д е л е н и е 3 . Мера $\pi_{0}$ с носителем на множестве чистых состояний и барицентром $\rho_{0}$ такая, что

$$
\chi_{\Phi}\left(\rho_{0}\right)=\int_{\mathfrak{S}(\mathscr{H})} H\left(\Phi(\sigma) \| \Phi\left(\rho_{0}\right)\right) \pi_{0}(d \sigma),
$$

называется $\chi_{\Phi}$-оптимальной мерой для состояния $\rho_{0}$.

Заметим, что из $\chi_{\Phi}(\rho)<+\infty$ не следует $H_{\Phi}(\rho)<+\infty$. Действительно, нетрудно построить канал $\Phi$ из конечномерного в бесконечномерное пространство такой, что $H_{\Phi}(\rho)=+\infty$ для любого состояния $\rho$ из $\mathfrak{S}(\mathscr{H}){ }^{3)}$ Но в силу свойства монотонности относительной энтро-

3) Например, канал $\Phi: \mathfrak{T}(\mathscr{H}) \ni A \mapsto \frac{1}{2} A \oplus \frac{1}{2} \sigma \operatorname{Tr} A \in \mathfrak{T}\left(\mathscr{H}^{\prime}\right)$, где $\sigma-$ фиксированное состояние с бесконечной энтропией. 
пии [23] имеем

$$
\sum_{i} \pi_{i} H\left(\Phi\left(\rho_{i}\right) \| \Phi(\rho)\right) \leqslant \sum_{i} \pi_{i} H\left(\rho_{i} \| \rho\right) \leqslant \log \operatorname{dim} \mathscr{H}<+\infty
$$

для любого ансамбля $\left\{\pi_{i}, \rho_{i}\right\}$, и, следовательно $\chi_{\Phi}(\rho) \leqslant \log \operatorname{dim} \mathscr{H}<+\infty$ для любого состояния $\rho$ из $\mathfrak{S}(\mathscr{H})$.

Из определения $\chi$-пропускной способности $\mathscr{A}$-ограниченного канала $\Phi$ следует, что $\bar{C}(\Phi, \mathscr{A})=\sup _{\rho \in \mathscr{A}} \chi_{\Phi}(\rho)$, причем, как показано в [10], вопрос о достижимости точной верхней грани в данном выражении тесно связан с существованием оптимальной меры для $\mathscr{A}$-ограниченного канала $\Phi$ и, как показывают примеры в [32], может иметь отрицательное решение.

Результаты предыдущего раздела позволяют доказать важное неравенство, определяющее поведение $\chi$-функции на любом выпуклом подмножестве состояний, конечномерная версия которого была получена в [21]. Это неравенство играет существенную роль в доказательстве теоремы 2 в разделе 7.

Предложение 3. Пусть $\Phi: \mathfrak{S}(\mathscr{H}) \mapsto \mathfrak{S}\left(\mathscr{H}^{\prime}\right)-$ произвольный канал, а $\mathscr{A}$ - выпуклое подмножество множества $\mathfrak{S}(\mathscr{H})$. Тогда для любого состояния $\rho$ из $\mathscr{A}$ имеет место неравенство

$$
\chi_{\Phi}(\rho) \leqslant \bar{C}(\Phi, \mathscr{A})-H(\Phi(\rho) \| \Omega(\Phi, \mathscr{A})) \leqslant \bar{C}(\Phi, \mathscr{A})-\frac{1}{2}\|\Phi(\rho)-\Omega(\Phi, \mathscr{A})\|_{1}^{2} .
$$

Д о к а з а т ел ь с т в о. Пусть $\bar{C}(\Phi, \mathscr{A})<+\infty,\left\{\pi_{i}, \rho_{i}\right\}-$ произвольный ансамбль такой, что $\sum_{i} \pi_{i} \rho_{i}=\rho$. В силу предложения 1

$$
\sum_{i} \pi_{i} H\left(\Phi\left(\rho_{i}\right) \| \Omega(\Phi, \mathscr{A})\right) \leqslant \bar{C}(\Phi, \mathscr{A}) .
$$

Из этого неравенства, тождества Дональда

$$
\sum_{i} \pi_{i} H\left(\Phi\left(\rho_{i}\right) \| \Omega(\Phi, \mathscr{A})\right)=\sum_{i} \pi_{i} H\left(\Phi\left(\rho_{i}\right) \| \Phi(\rho)\right)+H(\Phi(\rho) \| \Omega(\Phi, \mathscr{A}))
$$

и определения $\chi$-функции (14) следует первое неравенство предложения. Второе неравенство следует из неравенства (1). Предложение 3 доказано.

Для всякого состояния $\rho$ с конечной выходной энтропией $\chi$-функция имеет сіедующее представление:

$$
\chi_{\Phi}(\rho)=H_{\Phi}(\rho)-\operatorname{co} H_{\Phi}(\rho),
$$

где

$$
\operatorname{co} H_{\Phi}(\rho)=\inf _{\sum_{i} \pi_{i} \rho_{i}=\rho} \sum_{i} \pi_{i} H_{\Phi}\left(\rho_{i}\right)
$$

- выпуклая оболочка выходной энтропии (см. приложение A).

В конечномерном случае выходная энтропия $H_{\Phi}$ и ее выпуклая оболочка со $H_{\Phi}$ - непрерывные функции на $\mathfrak{S}(\mathscr{H})$, первая из которых вогнутая, а вторая - выпуклая, и представление (15) имеет место для 
всех состояний. Следовательно, в этом случае функция $\chi_{\Phi}$ непрерывна и вогнута на $\mathfrak{S}(\mathscr{H})$.

В бесконечномерном случае выходная энтропия $H_{\Phi}$ обладает лишь свойством полунепрерывности снизу, и, следовательно, функция $\chi_{\Phi}$ не является непрерывной даже в случае тождественного канала $\Phi$, для которого $\chi_{\Phi}=H_{\Phi}$. Следующее предложение показывает, что функция $\chi_{\Phi}$ для произвольного канала $\Phi$ обладает свойствами, аналогичными свойствам выходной энтропии $H_{\Phi}$.

Предложение 4. Для произвольного канала $\Phi$ функиия $\chi_{\Phi} я в л я-$ ется неотрииательной полунепрерывной снизу вогнутой функиией на $\mathfrak{S}(\mathscr{H})$, причем

$$
\chi_{\Phi}(\rho)-\sum_{i=1}^{n} \pi_{i} \chi_{\Phi}\left(\rho_{i}\right) \geqslant \sum_{i=1}^{n} \pi_{i} H\left(\Phi\left(\rho_{i}\right) \| \Phi(\rho)\right)
$$

для любого ансамбля состояний $\left\{\pi_{i}, \rho_{i}\right\}_{i=1}^{n}$ со средним состоянием $\rho$.

Неравенство (17) можно рассматривать как обобщение на случай $\chi$-функции известного тождества для квантовой энтропии

$$
H(\rho)-\sum_{i=1}^{n} \pi_{i} H\left(\rho_{i}\right)=\sum_{i=1}^{n} \pi_{i} H\left(\rho_{i} \| \rho\right) .
$$

Д о к а з а т е л ь с т в о. Неотрицательность $\chi$-функции очевидна. Полунепрерывность снизу доказана в [31] (предложение 3). Докажем неравенство (17), из которого следует вогнутость $\chi$-функции. По определению $\chi$-функции по заданному $\varepsilon>0$ для каждого $i=1, \ldots, n$ можно найти ансамбль $\left\{\mu_{j}^{i}, \sigma_{j}^{i}\right\}_{j=1}^{m(i)}$ со средним состоянием $\rho_{i}$ такой, что $\chi_{\Phi}\left(\left\{\mu_{j}^{i}, \sigma_{j}^{i}\right\}\right)>$ $\chi_{\Phi}\left(\rho_{i}\right)-\varepsilon$. Поскольку среднее состояние ансамбля $\sum_{i=1}^{n} \pi_{i}\left\{\mu_{j}^{i}, \sigma_{j}^{i}\right\}$ совпадает с $\rho$, используя лемму 1 , получаем

$$
\begin{aligned}
\chi_{\Phi}(\rho) & \geqslant \chi_{\Phi}\left(\sum_{i=1}^{n} \pi_{i}\left\{\mu_{j}^{i}, \sigma_{j}^{i}\right\}\right)=\sum_{i=1}^{n} \pi_{i} \chi_{\Phi}\left(\left\{\mu_{j}^{i}, \sigma_{j}^{i}\right\}\right)+\sum_{i=1}^{n} \pi_{i} H\left(\Phi\left(\rho_{i}\right) \| \Phi(\rho)\right) \\
& \geqslant \sum_{i=1}^{n} \pi_{i} \chi_{\Phi}\left(\rho_{i}\right)+\sum_{i=1}^{n} \pi_{i} H\left(\Phi\left(\rho_{i}\right) \| \Phi(\rho)\right)-\varepsilon .
\end{aligned}
$$

Из произвольности выбора $\varepsilon$ следует неравенство (17). Предложение 4 доказано.

В современном выпуклом анализе существенную роль играет понятие сильной выпуклости (вогнутости) [6]. С учетом неравенства (1) из предложения 4 вытекает следующее наблюдение.

Следствие 2. Для любого канала Ф функиия $\chi_{\Phi}$ является сильно вогнутой функцией на $\mathfrak{S}(\mathscr{H})$ в следующем смьсле:

$\chi_{\Phi}\left(\lambda \rho_{1}+(1-\lambda) \rho_{2}\right) \geqslant \lambda \chi_{\Phi}\left(\rho_{1}\right)+(1-\lambda) \chi_{\Phi}\left(\rho_{2}\right)+\frac{1}{2} \lambda(1-\lambda)\left\|\Phi\left(\bar{\rho}_{2}\right)-\Phi\left(\bar{\rho}_{1}\right)\right\|_{1}^{2}$ для любых состояний $\rho_{1}$ и $\rho_{2}$ из $\mathfrak{S}(\mathscr{H})$ и любого $\lambda$ из $[0,1]$. 
Сходство свойств функций $\chi_{\Phi}$ и $H_{\Phi}$ усиливает следующий аналог теоремы Саймона о мажорируемой сходимости [33]. ${ }^{4)}$

Следствие 3. Пусть $\left\{\rho_{n}\right\}$ - последовательность состояний из $\mathfrak{S}(\mathscr{H})$, сходящаяся $\kappa$ состоянию $\rho_{0}$, такая, что $\lambda_{n} \rho_{n} \leqslant \rho_{0}$ для некоторой последовательности $\left\{\lambda_{n}\right\}$ положительных чисел, сходящейся $\kappa 1$. Тогда

$$
\lim _{n \rightarrow+\infty} \chi_{\Phi}\left(\rho_{n}\right)=\chi_{\Phi}\left(\rho_{0}\right) .
$$

Д о к а з а т е л с с в о. Из условия $\lambda_{n} \rho_{n} \leqslant \rho_{0}$ следует разложение $\rho_{0}=\lambda_{n} \rho_{n}+\left(1-\lambda_{n}\right) \sigma_{n}$, где $\sigma_{n}=\left(1-\lambda_{n}\right)^{-1}\left(\rho_{0}-\lambda_{n} \rho_{n}\right)-$ некоторое состояние из $\mathfrak{S}(\mathscr{H})$. В силу вогнутости $\chi$-функции имеем

$$
\chi_{\Phi}\left(\rho_{0}\right) \geqslant \lambda_{n} \chi_{\Phi}\left(\rho_{n}\right)+\left(1-\lambda_{n}\right) \chi_{\Phi}\left(\sigma_{n}\right) \geqslant \lambda_{n} \chi_{\Phi}\left(\rho_{n}\right) \quad \forall n,
$$

и, следовательно, $\lim \sup _{n \rightarrow+\infty} \chi_{\Phi}\left(\rho_{n}\right) \leqslant \chi_{\Phi}\left(\rho_{0}\right)$. Из полунепрерывности снизу $\chi$-функции вытекает существование указанного выше предела. Следствие 3 доказано.

3 а м е ч ан и е 2. Следствие 3 позволяет аппроксимировать значение $\chi_{\Phi}\left(\rho_{0}\right)$ для произвольного состояния $\rho_{0}$ последовательностью $\left\{\chi_{\Phi}\left(\rho_{n}\right)\right\}$, в которой $\rho_{n}=\left(\operatorname{Tr} P_{n} \rho\right)^{-1} P_{n} \rho_{0}$ - состояние конечного ранга при каждом $n$, где $P_{n}$ - спектральный проектор состояния $\rho_{0}$, соответствующий $n$ наибольшим собственным значениям.

Полученные в следующем разделе свойства выпуклого замыкания выходной энтропии позволят доказать, что $\chi$-функция имеет непрерывные сужения на любое подмножество состояний, на котором непрерывна выходная энтропия (теорема 1).

Отметим следующие цепные свойства $\chi$-функции.

Предложение 5. Пусть $\Phi: \mathfrak{S}(\mathscr{H}) \mapsto \mathfrak{S}\left(\mathscr{H}^{\prime}\right)$ u $\Psi: \mathfrak{S}\left(\mathscr{H}^{\prime}\right) \mapsto$ $\mathfrak{S}\left(\mathscr{H}^{\prime \prime}\right)$ - два произвольньх канала. Тогда

$$
\chi_{\Psi \circ \Phi}(\rho) \leqslant \chi_{\Phi}(\rho) \quad \text { и } \quad \chi_{\Psi \circ \Phi}(\rho) \leqslant \chi_{\Psi}(\Phi(\rho)) \quad \text { для всех } \rho \text { из } \mathfrak{S}(\mathscr{H}) .
$$

Д о к а з а т е л ь т в о. Первое неравенство следует из свойства монотонности относительной энтропии [23] и определения (14), второе является прямым следствием определения (14).

5. Выпуклое замыкание выходной энтропии. В конечномерном случае выходная энтропия $H_{\Phi}$ конечна и функция $\chi_{\Phi}$ представима выражением (15) как разность между выходной энтропией $H_{\Phi}$ и ее выпуклой оболочкой со $H_{\Phi}$. В этом случае функция со $H_{\Phi}$ непрерывна и, следовательно, замкнута (см. приложение А). Это означает, что выпуклая оболочка со $H_{\Phi}$ выходной энтропии совпадает с выпуклым замыканием $\overline{\mathrm{co}} H_{\Phi}$ выходной энтропии.

4) Эту теорему можно сформулировать также как следствие 3 с заменой функции $\chi_{\Phi}$ на квантовую энтропию $H$. 
В бесконечномерном случае функция со $H_{\Phi}$ не является замкнутой даже в случае тождественного канала $\Phi$. Действительно, со $H_{\Phi}(\rho)=+\infty$ для любого состояния $\rho$ с $H_{\Phi}(\rho)=+\infty$ (см. доказательство леммы 2), но такое состояние $\rho$ является пределом последовательности $\left\{\rho_{n}\right\}$ состояний конечного ранга, для которых со $H_{\Phi}\left(\rho_{n}\right)=0$. Следовательно, функция со $H_{\Phi}$ не является полунепрерывной снизу.

Естественно предположить, что в бесконечномерном случае роль функции со $H_{\Phi}$ играет функция со $H_{\Phi}$. Цель данного раздела - подтвердить это предположение посредством исследования свойств функции со $H_{\Phi}$ и ее связи с $\chi$-функцией.

Прежде всего получим явное представление для со $H_{\Phi}$. Рассмотрим функцию

$$
\widehat{H}_{\Phi}(\rho)=\inf _{\pi \in \mathscr{P}_{\{\rho\}}} \int_{\mathfrak{S}(\mathscr{H})} H_{\Phi}(\rho) \pi(d \rho) \leqslant+\infty
$$

где $\mathscr{P}_{\{\rho\}}-$ множество всех вероятностных мер с барицентром $\rho$. Ясно, что $\widehat{H}_{\Phi}(\rho) \leqslant$ со $H_{\Phi}(\rho) \leqslant H_{\Phi}(\rho)$ для всех состояний $\rho$ из $\mathfrak{S}(\mathscr{H})$. Исследуя свойства функции $\hat{H}_{\Phi}$, мы установим в дальнейшем, что $\widehat{H}_{\Phi}=\overline{\text { со }} H_{\Phi}$ (предложение 7).

Как отмечалось в предыдущем разделе, в определении $\chi$-функции точная верхняя грань по всем мерам совпадает с точной верхней гранью по всем мерам с конечным носителем (обычным ансамблям). В случае $\widehat{H}$-функции имеет место другая ситуация.

Лемма 2. Равенство $\widehat{H}_{\Phi}(\rho)=\inf _{\sum_{i} \pi_{i} \rho_{i}=\rho} \sum_{i} \pi_{i} H_{\Phi}\left(\rho_{i}\right)=\operatorname{co} H_{\Phi}(\rho)$ имеет место тогда и только тогда, когда либо $H_{\Phi}(\rho)<+\infty$, либо $\widehat{H}_{\Phi}(\rho)=+\infty$.

Д о к а з а т е л ь с т в о. Если $H_{\Phi}(\rho)<+\infty$, то $\chi_{\Phi}(\rho)=H_{\Phi}(\rho)-$ со $H_{\Phi}(\rho)$. В силу [10, предложение 1 и следствие 1$]$ имеем $\chi_{\Phi}(\rho)=H_{\Phi}(\rho)-$ $\widehat{H}_{\Phi}(\rho)$ и, следовательно, $\widehat{H}_{\Phi}(\rho)=\operatorname{co} H_{\Phi}(\rho)$.

Если $H_{\Phi}(\rho)=+\infty$, то со $H_{\Phi}(\rho)=+\infty$, поскольку множество состояний с конечной выходной энтропией выпукло [34]. Лемма 2 доказана.

Из леммы 2 вытекает, что $\widehat{H}_{\Phi}(\rho)<\operatorname{co} H_{\Phi}(\rho)$ для любого состояния $\rho$ такого, что $H_{\Phi}(\rho)=+\infty$ и $\widehat{H}_{\Phi}(\rho)<+\infty$. Заметим, что множество таких состояний непусто. Например, в случае тождественного канала $\Phi$ нетрудно видеть, что $\widehat{H}_{\Phi}(\rho)=0$ для любого состояния $\rho$, но множество всех состояний $\rho$, для которых $H_{\Phi}(\rho)<+\infty$, является подмножеством первой категории в множестве всех состояний $\mathfrak{S}(\mathscr{H})$ [34].

Покажем прежде всего, что точную нижнюю грань в определении $\widehat{H}_{\Phi}(\rho)$ можно брать только по мерам с носителем на множестве чистых состояний. Для этого рассмотрим следующее отношение частичного порядка на множестве $\mathscr{P}$. Пусть $\mathscr{S}-$ множество всех выпуклых непрерывных ограниченных функций на $\mathfrak{S}(\mathscr{H})$. Положим $\mu \succ \nu$ тогда 
и только тогда, когда

$$
\int_{\mathfrak{S}(\mathscr{H})} f(\rho) \mu(d \rho) \geqslant \int_{\mathfrak{S}(\mathscr{H})} f(\rho) \nu(d \rho) \quad \text { для всех } f \text { из } \mathscr{S} .
$$

Такое отношение частичного порядка на множествах вероятностных мер, часто называемое порядком Шоке (the Choquet ordering), подробно изучено (см., например, [14]).

Предложение 6. Для любого состояния $\rho_{0}$ существует мера $\pi_{0} c$ баричентром $\rho_{0}$ и носителем на множестве чистьх состояний такая, umo

$$
\widehat{H}_{\Phi}\left(\rho_{0}\right)=\int_{\mathfrak{S}(\mathscr{H})} H_{\Phi}(\rho) \pi_{0}(d \rho)
$$

$B$ качестве $\pi_{0}$ можно вибрать меру с конечньм носителем, состояшим из $n^{2}$ атомов (ансамбль из $n^{2}$ чистых состояний) тогда и только тогда, когда состояние $\rho_{0}$ имеет конечный ранг $n$.

Д о к а з а т е л ь с т в о. В доказательстве теоремы в [10] показано, что функционал

$$
\pi \mapsto \int_{\mathfrak{S}(\mathscr{H})} H_{\Phi}(\rho) \pi(d \rho)
$$

корректно определен и полунепрерывен снизу на множестве $\mathscr{P}$ с топологией слабой сходимости. В силу предложения 2 из [10] множество $\mathscr{P}_{\left\{\rho_{0}\right\}}$ компактно в этой топологии. Следовательно, данный функционал достигает своего минимума на множестве $\mathscr{P}_{\left\{\rho_{0}\right\}}$ на некоторых $\operatorname{mepax} \pi_{*} \in \mathscr{P}_{\left\{\rho_{0}\right\}}$, т.е.

$$
\widehat{H}_{\Phi}\left(\rho_{0}\right)=\int_{\mathfrak{S}(\mathscr{H})} H_{\Phi}(\rho) \pi_{*}(d \rho)
$$

Для доказательства того, что среди всех таких мер $\pi_{*}$ найдется мера $\pi_{0}$ с носителем на множестве чистых состояний, нам потребуются два простых свойства введенного выше отношения порядка.

1. Пусть $\left\{\mu_{n}\right\}$ u $\left\{\nu_{n}\right\}-$ две последовательности из Я尹, слабо сходящиеся $\kappa$ мерам $\mu$ и соответственно $и$ такие, что $\mu_{n} \succ \nu_{n} \partial \Omega я$ всех $n$. Тогда $\mu \succ \nu$.

2. Если $\mu \succ \nu$, mo

$$
\int_{\mathfrak{S}(\mathscr{H})} g(\rho) \mu(d \rho) \geqslant \int_{\mathfrak{S}(\mathscr{H})} g(\rho) \nu(d \rho)
$$

для любой Функиии $g$, которая представима как (поточечньй) предел монотонной последовательности функиий из $\mathscr{S}$.

В силу леммы 1 из [10] существует последовательность $\left\{\pi_{n}\right\}$ мер из $\mathscr{P}_{\left\{\rho_{0}\right\}}$ с конечным носителем, слабо сходящаяся к мере $\pi_{*}$. Представляя каждый атом меры $\pi_{n}$ в виде выпуклой комбинации чистых состояний, мы получим меру $\widehat{\pi}_{n}$ с тем же барицентром и счетным носителем на множестве чистых состояний. Легко видеть, что $\widehat{\pi}_{n} \succ \pi_{n}$. В силу 
упомянутой выше компактности множества $\mathscr{P}_{\left\{\rho_{0}\right\}}$ существует подпоследовательность $\left\{\widehat{\pi}_{n_{k}}\right\}$, сходящаяся к некоторой мере $\pi_{0}$. В силу замкнутости множества чистых состояний и теоремы 6.1 из [28] носитель этой меры $\pi_{0}$ сосредоточен на чистых состояниях. Поскольку $\widehat{\pi}_{n_{k}} \succ \pi_{n_{k}}$ для всех $k$, из свойства 1 частичного порядка $\succ$ следует $\pi_{0} \succ \pi_{*}$.

В силу леммы 4 из [22] выпуклая функция $g(\rho)=-H_{\Phi}(\rho)=$ $-H(\Phi(\rho))$ является поточечным пределом монотонной последовательности ограниченных непрерывных функций

$$
g_{n}(\rho)=-\left(\operatorname{Tr} P_{n} \Phi(\rho)\right) H\left(\left(\operatorname{Tr} P_{n} \Phi(\rho)\right)^{-1} P_{n} \Phi(\rho) P_{n}\right),
$$

где $\left\{P_{n}\right\}$ - произвольная возрастающая последовательность проекторов конечного ранга, сильно сходящаяся к тождественному оператору $I_{\mathscr{H}}$. Нетрудно видеть, что при каждом $n$ функция $g_{n}$ выпукла и, следовательно, лежит в $\mathscr{S}$. Используя свойство 2 частичного порядка $\succ$, с учетом (19) получаем

$$
\widehat{H}_{\Phi}\left(\rho_{0}\right)=\int_{\mathfrak{S}(\mathscr{H})} H_{\Phi}(\rho) \pi_{*}(d \rho) \geqslant \int_{\mathfrak{S}(\mathscr{H})} H_{\Phi}(\rho) \pi_{0}(d \rho) .
$$

По определению функции $\widehat{H}_{\Phi}$ последнее неравенство является равенством.

Докажем последнее утверждение предложения. Если состояние $\rho_{0}$ имеет бесконечный ранг, то множество $\mathscr{P}_{\left\{\rho_{0}\right\}}$ не содержит мер с конечным носителем на множестве чистых состояний.

Пусть состояние $\rho_{0}$ имеет конечный ранг $n, \mathscr{H}_{0}=\operatorname{supp} \rho_{0}-$ $n$-мерное подпространство и $\Phi_{0}$ - подканал канала $\Phi$, соответствующий подпространству $\mathscr{H}_{0}$ (подканалом $\Phi_{0}$ канала $\Phi$, соответствующим подпространству $\mathscr{H}_{0}$, называется сужение канала $\Phi$ на множество состояний, носители которых лежат в подпространстве $\left.\mathscr{H}_{0}[31]\right)$.

Если $H_{\Phi_{0}}\left(\rho_{0}\right)=H_{\Phi}\left(\rho_{0}\right)<+\infty$, то, в силу приведенной ниже леммы 3 , функция $H_{\Phi_{0}}$ непрерывна на компактном множестве $\mathfrak{S}\left(\mathscr{H}_{0}\right)$. Это позволяет применить лемму А-2 из [35] для доказательства существования ансамбля из $\left(\operatorname{dim} \mathscr{H}_{0}\right)^{2}$ состояний со средним $\rho_{0}$, на котором достигается точная нижняя грань в определении функции со $H_{\Phi_{0}}$, совпадающей с сужением функции со $H_{\Phi}$ на подмножество $\mathfrak{S}\left(\mathscr{H}_{0}\right)$ множества $\mathfrak{S}(\mathscr{H})$. В силу леммы 2 сужение функции со $H_{\Phi}$ на множество $\mathfrak{S}\left(\mathscr{H}_{0}\right)$ совпадает с сужением на это множество функции $\widehat{H}_{\Phi}$.

Если $H_{\Phi_{0}}\left(\rho_{0}\right)=H_{\Phi}\left(\rho_{0}\right)=+\infty$, то $\widehat{H}_{\Phi}\left(\rho_{0}\right)=+\infty$ и, следовательно, любой ансамбль со средним состоянием $\rho_{0}$ является оптимальным. Для доказательства этого утверждения заметим, что из $H_{\Phi}\left(\rho_{0}\right)=+\infty$ следует $H_{\Phi}(\sigma)=+\infty$ для любого состояния $\sigma$ такого, что $\operatorname{supp} \sigma=$ $\operatorname{supp} \rho_{0}=\mathscr{H}_{0}$. Действительно, для этого состояния $\sigma$ существует положительное число $\lambda_{\sigma}$ такое, что $\lambda_{\sigma} \sigma \geqslant \rho_{0}$. В силу неотрицательности относительной энтропии имеем $\lambda_{\sigma} \operatorname{Tr} \Phi(\sigma)(-\log \Phi(\sigma)) \geqslant \operatorname{Tr} \Phi\left(\rho_{0}\right)(-\log \Phi(\sigma)) \geqslant \operatorname{Tr} \Phi\left(\rho_{0}\right)\left(-\log \Phi\left(\rho_{0}\right)\right)=+\infty$. 
Предположим, что $\widehat{H}_{\Phi}\left(\rho_{0}\right)<+\infty$. Тогда существует мера $\pi$ с барицентром $\rho_{0}$ такая, что функция $H_{\Phi}$ конечна $\pi$-почти всюду. Пусть $\mathscr{F}$ - подмножество $\mathfrak{S}\left(\mathscr{H}_{0}\right)$ такое, что функция $H_{\Phi}$ конечна на множестве $\mathscr{F}$ и $\pi(\mathscr{F})=1$. Из равенства $\rho_{0}=\int_{\mathscr{F}} \rho \pi(d \rho)$ следует, что линейная оболочка подпространств $\{\operatorname{supp} \rho\}_{\rho \in \mathscr{F}}$ совпадает с $\mathscr{H}_{0}$ и, следовательно, существует конечный набор $\left\{\rho_{i}\right\}_{i=1}^{n}$ состояний из $\mathscr{F}$ таких, что $\operatorname{supp}\left(n^{-1} \sum_{i=1}^{n} \rho_{i}\right)=\mathscr{H}_{0}$. Поскольку состояние $n^{-1} \sum_{i=1}^{n} \rho_{i}$ есть конечная выпуклая комбинация состояний $\rho_{i}, i=1, \ldots, n$, у которых $H_{\Phi}\left(\rho_{i}\right)<+\infty$ для всех $i=1, \ldots, n$, заключаем, что $H_{\Phi}\left(n^{-1} \sum_{i=1}^{n} \rho_{i}\right)<+\infty$ [34]. Но это противоречит предыдущему наблюдению. Предложение 6 доказано.

Лемма 3. Пусть $\Phi: \mathfrak{S}(\mathscr{H}) \mapsto \mathfrak{S}\left(\mathscr{H}^{\prime}\right)$ - канал такой, что $\operatorname{dim} \mathscr{H}<+\infty$. Eсли существует состояние полного ранга $\rho_{0}$ такое, что $H_{\Phi}\left(\rho_{0}\right)<+\infty$, то функция $H_{\Phi}$ непрерывна на множестве $\mathfrak{S}(\mathscr{H})$.

Д о к а з а т е л ь с т в о. Пусть $I_{\mathscr{H}}$ - тождественный оператор в пространстве $\mathscr{H}$. Из конечномерности $\mathscr{H}$ следует, что $\lambda I_{\mathscr{H}} \leqslant \rho_{0}$ для некоторого положительного $\lambda$, и поэтому $H_{\Phi}\left(I_{\mathscr{H}}\right)<+\infty$. Утверждение леммы следует из теоремы Саймона о мажорируемой сходимости [33] (с использованием $\Phi\left(I_{\mathscr{H}}\right)$ в качестве оператора $\left.B\right)$.

О п р е д е л ен и е 4 . Мера $\pi_{0}$ с указанными в предложении 6 свойствами называется $\widehat{H}_{\Phi}$-оптимальной мерой для состояния $\rho_{0}$.

Легко видеть, что множество $\widehat{H}_{\Phi}$-оптимальных мер совпадает со множеством $\chi_{\Phi}$-оптимальных мер для любого состояния $\rho_{0}$ с конечной выходной энтропией $H_{\Phi}\left(\rho_{0}\right)$.

Другое важное свойство функции $\widehat{H}_{\Phi}(\rho)$ установлено в следующей лемме.

Лемма 4. Функиия $\widehat{H}_{\Phi}(\rho)$ выпукла и полунепрерывна снизу на $\mathfrak{S}(\mathscr{H})$.

Д ок а з а тель с т о. Для доказательства выпуклости функции $\widehat{H}_{\Phi}$ достаточно заметить, что

$$
\lambda \mathscr{P}_{\left\{\rho_{1}\right\}}+(1-\lambda) \mathscr{P}_{\left\{\rho_{2}\right\}} \subseteq \mathscr{P}_{\left\{\lambda \rho_{1}+(1-\lambda) \rho_{2}\right\}}
$$

для любых состояний $\rho_{1}, \rho_{2}$ и числа $\lambda$ из $[0,1]$.

Предположим, что функция $\widehat{H}_{\Phi}$ не является полунепрерывной снизу. Это означает существование последовательности состояний $\left\{\rho_{n}\right\}$, сходящейся к некоторому состоянию $\rho_{0}$, такой, что

$$
\lim _{n \rightarrow+\infty} \widehat{H}_{\Phi}\left(\rho_{n}\right)<\widehat{H}_{\Phi}\left(\rho_{0}\right) .
$$

В силу предложения 6 для каждого $n=1,2, \ldots$ сушествует мера $\pi_{n}$ из $\mathscr{P}_{\left\{\rho_{n}\right\}}$ такая, что

$$
\widehat{H}_{\Phi}\left(\rho_{n}\right)=\int_{\mathfrak{S}(\mathscr{H})} H_{\Phi}(\rho) \pi_{n}(d \rho) .
$$


Множество $\mathscr{A}=\left\{\rho_{n}\right\}_{n=0}^{+\infty}$ является компактным подмножеством $\mathfrak{S}(\mathscr{H})$. В силу предложения 2 из [10] множество $\mathscr{P}_{\mathscr{A}}$ компактно. Поскольку $\left\{\pi_{n}\right\} \subset \mathscr{P}_{\mathscr{A}}$, сушествует подпоследовательность $\left\{\pi_{n_{k}}\right\}$, сходяшаяся к некоторой мере $\pi_{0}$. Из непрерывности отображения $\pi \mapsto \bar{\rho}(\pi)$ вытекает $\pi_{0} \in \mathscr{P}_{\left\{\rho_{0}\right\}}$. В силу полунепрерывности снизу функционала (18) получаем

$$
\begin{aligned}
\widehat{H}_{\Phi}\left(\rho_{0}\right) & \leqslant \int_{\mathfrak{S}(\mathscr{H})} H_{\Phi}(\rho) \pi_{0}(d \rho) \leqslant \liminf _{k \rightarrow+\infty} \int_{\mathfrak{S}(\mathscr{H})} H_{\Phi}(\rho) \pi_{n_{k}}(d \rho) \\
& =\lim _{k \rightarrow+\infty} \widehat{H}_{\Phi}\left(\rho_{n_{k}}\right)
\end{aligned}
$$

что противоречит (20). Лемма 4 доказана.

Предложение 7. Функиия $\widehat{H}_{\Phi}$ совпадает с выпукльмм замыканием ${ }^{5)} \overline{\mathrm{co}} H_{\Phi}$ вьхходной энтропии $H_{\Phi}$, причем если $\overline{\mathrm{co}} H_{\Phi}(\rho)<+\infty$, то

$$
\left\{\overline{\mathrm{co}} H_{\Phi}(\rho)=\operatorname{co} H_{\Phi}(\rho)\right\} \Longleftrightarrow\left\{H_{\Phi}(\rho)<+\infty\right\} .
$$

Д о к а з а т е л ь с т в о. Из леммы 4 и определения выпуклого замыкания следует, что

$$
\widehat{H}_{\Phi}(\rho) \leqslant \overline{\operatorname{co}} H_{\Phi}(\rho) \leqslant \operatorname{co} H_{\Phi}(\rho) \leqslant H_{\Phi}(\rho) \quad \forall \rho \in \mathfrak{S}(\mathscr{H}) .
$$

В силу леммы $2 \widehat{H}_{\Phi}\left(\rho_{0}\right)$ совпадает с со $H_{\Phi}\left(\rho_{0}\right)$ для любого состояния $\rho_{0}$ с конечной выходной энтропией $H_{\Phi}\left(\rho_{0}\right)$. Поэтому $(21)$ означает, что $\widehat{H}_{\Phi}\left(\rho_{0}\right)=\overline{\mathrm{co}} H_{\Phi}\left(\rho_{0}\right)$ для всех таких состояний.

Пусть $\rho_{0}-$ произвольное состояние такое, что $\widehat{H}_{\Phi}\left(\rho_{0}\right)<+\infty$. В силу приводимой ниже леммы 5 существует последовательность $\left\{\rho_{n}\right\}$ состояний с конечной выходной энтропией, сходящаяся к состоянию $\rho_{0}$, такая, что $\lim _{n \rightarrow+\infty} \widehat{H}_{\Phi}\left(\rho_{n}\right)=\widehat{H}_{\Phi}\left(\rho_{0}\right)$. Как показано выше, $\widehat{H}_{\Phi}\left(\rho_{n}\right)=$ $\overline{\mathrm{co}} H_{\Phi}\left(\rho_{n}\right)$ для всех $n$. Поскольку функция со $H_{\Phi}$ полунепрерывна снизу (по определению), получаем

$$
\overline{\mathrm{CO}} H_{\Phi}\left(\rho_{0}\right) \leqslant \liminf _{n \rightarrow+\infty} \overline{\mathrm{co}} H_{\Phi}\left(\rho_{n}\right)=\lim _{n \rightarrow+\infty} \widehat{H}_{\Phi}\left(\rho_{n}\right)=\widehat{H}_{\Phi}\left(\rho_{0}\right) .
$$

Из этого неравенства и из $(21)$ следует, что $\widehat{H}_{\Phi}\left(\rho_{0}\right)=\overline{\mathrm{co}} H_{\Phi}\left(\rho_{0}\right)$ для произвольного состояния $\rho_{0}$. Предложение 7 доказано.

При изучении свойств функции $\widehat{H}_{\Phi}$ важную роль играет следующее наблюдение.

Лемма 5. Для любого состояния $\rho_{0}$ такого, что $\widehat{H}_{\Phi}\left(\rho_{0}\right)<\infty$, существует последовательность $\left\{\rho_{n}\right\}$ состояний конечного ранга, сходящаяся $\mathcal{\kappa}$ состоянию $\rho_{0}$, такая, что

$$
H_{\Phi}\left(\rho_{n}\right)<+\infty \quad \text { для всех } n \quad u \quad \lim _{n \rightarrow+\infty} \widehat{H}_{\Phi}\left(\rho_{n}\right)=\widehat{H}_{\Phi}\left(\rho_{0}\right) .
$$

\footnotetext{
5) см. приложение А.
} 


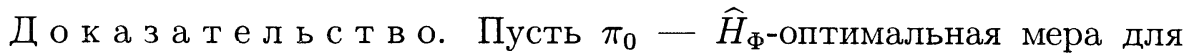
состояния $\rho_{0}$, существующая по предложению 6 . Поскольку всякая вероятностная мера на полном сепарабельном метрическом пространстве $\mathfrak{S}(\mathscr{H})$ является плотной [1], [28], для любого $n \in \mathbf{N}$ существует компактное подмножество $\mathscr{K}_{n}$ множества $\operatorname{Extr}(\mathfrak{S}(\mathscr{H}))$ такое, что $\pi_{0}\left(\mathscr{K}_{n}\right)>1-1 / n$. Из компактности множества $\mathscr{K}_{n}$ следует разложение $\mathscr{K}_{n}=\bigcup_{i=1}^{m(n)} \mathscr{A}_{i}^{n}$, где $\left\{\mathscr{A}_{i}^{n}\right\}_{i=1}^{m(n)}-$ конечный набор непересекающихся измеримых множеств с диаметром, не превосходящим $1 / n$. Без ограничения общности можно считать, что $\pi_{0}\left(\mathscr{A}_{i}^{n}\right)>0$ для всех $i$ и $n$. По построению компактное множество $\overline{\mathscr{A}_{i}^{n}}$ содержится в некотором шаре $\mathscr{B}_{i}^{n}$ диаметра $1 / n$ для всех $i$ и $n$.

По условию $\widehat{H}_{\Phi}\left(\rho_{0}\right)=\int_{\mathfrak{S}(\mathscr{H})} H_{\Phi}(\rho) \pi_{0}(d \rho)<+\infty$, и, следовательно, функция $H_{\Phi}$ конечна $\pi_{0}$-почти всюду. Поскольку функция $H_{\Phi}$ полунепрерывна снизу, она достигает своего конечного минимума на компактном множестве $\overline{\mathscr{A}_{i}^{n}}$ положительной меры в некотором состоянии $\rho_{i}^{n} \in \overline{\mathscr{A}_{i}^{n}}$. Рассмотрим состояние $\rho_{n}=\left(\pi_{0}\left(\mathscr{K}_{n}\right)\right)^{-1} \sum_{i=1}^{m(n)} \pi_{0}\left(\mathscr{A}_{i}^{n}\right) \rho_{i}^{n}$.

Покажем, что

$$
\left\|\rho_{n}-\rho_{0}\right\|_{1} \leqslant \frac{3}{n}
$$

Состояние $\widehat{\rho}_{i}^{n}=\left(\pi_{0}\left(\mathscr{A}_{i}^{n}\right)\right)^{-1} \int_{\mathscr{A}_{i}^{n}} \rho \pi_{0}(d \rho)$ лежит в $\mathscr{B}_{i}^{n}$ в силу выпуклости $\mathscr{B}_{i}^{n}$. Следовательно, $\left\|\rho_{i}^{n}-\widehat{\rho}_{i}^{n}\right\|_{1} \leqslant 1 / n$. Замечая, что $\pi_{0}\left(\mathscr{K}_{n}\right)=$ $\sum_{i=1}^{m(n)} \pi_{0}\left(\mathscr{A}_{i}^{n}\right)$, получаем

$$
\begin{aligned}
\left\|\rho_{n}-\rho_{0}\right\|_{1}= & \|\left(\pi_{0}\left(\mathscr{K}_{n}\right)\right)^{-1} \sum_{i=1}^{m(n)} \pi_{0}\left(\mathscr{A}_{i}^{n}\right) \rho_{i}^{n} \\
& -\sum_{i=1}^{m(n)} \int_{\mathscr{A}_{i}^{n}} \rho \pi_{0}(d \rho)-\int_{\mathfrak{S}(\mathscr{H}) \backslash \mathscr{K}_{n}} \rho \pi_{0}(d \rho) \|_{1} \\
\leqslant & \sum_{i=1}^{m(n)} \pi_{0}\left(\mathscr{A}_{i}^{n}\right)\left\|\left(\pi_{0}\left(\mathscr{K}_{n}\right)\right)^{-1} \rho_{i}^{n}-\widehat{\rho}_{i}^{n}\right\|_{1}+\left\|\int_{\mathfrak{S}(\mathscr{H}) \backslash \mathscr{K}_{n}} \rho \pi_{0}(d \rho)\right\|_{1} \\
\leqslant & \left(1-\pi_{0}\left(\mathscr{K}_{n}\right)\right)+\sum_{i=1}^{m(n)} \pi_{0}\left(\mathscr{A}_{i}^{n}\right)\left\|\rho_{i}^{n}-\widehat{\rho}_{i}^{n}\right\|_{1}+\pi_{0}\left(\mathfrak{S}(\mathscr{H}) \backslash \mathscr{K}_{n}\right)<\frac{3}{n},
\end{aligned}
$$

T.e. (22).

В силу выбора состояния $\rho_{i}^{n}$ при каждом $i$ и $n$ имеем $H_{\Phi}\left(\rho_{i}^{n}\right) \leqslant H_{\Phi}(\rho)$ для всех $\rho$ из $\mathscr{A}_{i}^{n}$. Следовательно,

$$
\begin{aligned}
\widehat{H}_{\Phi}\left(\rho_{n}\right) & \leqslant\left(\pi_{0}\left(\mathscr{K}_{n}\right)\right)^{-1} \sum_{i=1}^{m(n)} \pi_{0}\left(\mathscr{A}_{i}^{n}\right) H_{\Phi}\left(\rho_{i}^{n}\right) \\
& \leqslant\left(\pi_{0}\left(\mathscr{K}_{n}\right)\right)^{-1} \sum_{i=1}^{m(n)} \int_{\mathscr{A}_{i}^{n}} H_{\Phi}(\rho) \pi_{0}(d \rho) \\
& \leqslant\left(\pi_{0}\left(\mathscr{K}_{n}\right)\right)^{-1} \int_{\mathfrak{S}(\mathscr{H})} H_{\Phi}(\rho) \pi_{0}(d \rho)=\left(\pi_{0}\left(\mathscr{K}_{n}\right)\right)^{-1} \widehat{H}_{\Phi}\left(\rho_{0}\right) .
\end{aligned}
$$


Поэтому $\lim \sup _{n \rightarrow+\infty} \widehat{H}_{\Phi}\left(\rho_{n}\right) \leqslant \widehat{H}_{\Phi}\left(\rho_{0}\right)$. Но $\lim _{n \rightarrow+\infty} \rho_{n}=\rho_{0}$ в силу $(22)$, и, учитывая лемму 4 , получаем $\liminf _{n \rightarrow+\infty} \widehat{H}_{\Phi}\left(\rho_{n}\right) \geqslant \widehat{H}_{\Phi}\left(\rho_{0}\right)$. Следовательно, существует $\lim _{n \rightarrow+\infty} \widehat{H}_{\Phi}\left(\rho_{n}\right)=\widehat{H}_{\Phi}\left(\rho_{0}\right)$.

По построению состояние $\rho_{n}$ при каждом $n$ есть конечная выпуклая комбинация чистых состояний $\rho_{i}^{n}, i=1, \ldots, m(n)$, с конечной выходной энтропией $H_{\Phi}\left(\rho_{i}^{n}\right)$. Это означает, что $H_{\Phi}\left(\rho_{n}\right)<+\infty$ для всех $n$ [34]. Лемма 5 доказана.

Вещественное банахово пространство $\mathfrak{B}_{h}(\mathscr{H})$ всех эрмитовых операторов двойственно пространству $\mathfrak{T}_{h}(\mathscr{H})$ всех эрмитовых ядерных операторов. Неотрицательную полунепрерывную снизу функцию $H_{\Phi}$ на $\mathfrak{S}(\mathscr{H})$ можно расширить до неотрицательной полунепрерывной снизу функции $\bar{H}_{\Phi}$ на $\mathfrak{T}_{h}(\mathscr{H})$, полагая ее равной $+\infty$ на $\mathfrak{T}_{h}(\mathscr{H}) \backslash \mathfrak{S}(\mathscr{H})$. Преобразование Фенхеля функции $\bar{H}_{\Phi}$ (см. приложение А) определено на множестве $\mathfrak{B}_{h}(\mathscr{H})$ выражением

$$
H_{\Phi}^{*}(A)=\sup _{\rho \in \mathfrak{T}_{h}(\mathscr{H})}\left(\operatorname{Tr} A \rho-\bar{H}_{\Phi}(\rho)\right)=\sup _{\rho \in \mathfrak{S}(\mathscr{H})}\left(\operatorname{Tr} A \rho-H_{\Phi}(\rho)\right) .
$$

Двойное преобразование Фенхеля $H_{\Phi}^{* *}$ определено на множестве $\mathfrak{T}_{h}(\mathscr{H})$ выражением

$$
H_{\Phi}^{* *}(\rho)=\sup _{A \in \mathfrak{B}_{h}(\mathscr{H})}\left(\operatorname{Tr} A \rho-H_{\Phi}^{*}(A)\right) .
$$

Поскольку функция $\bar{H}_{\Phi}$ неотрицательна, ее выпуклое замыкание $\overline{\text { со }} \bar{H}_{\Phi}$ совпадает с двойным преобразованием Фенхеля $H_{\Phi}^{* *}$. Замечая, что сужение функции со $\bar{H}_{\Phi}$ на множество $\mathfrak{S}(\mathscr{H})$ совпадает с со $H_{\Phi}$, получаем, с учетом предложения 7 , следующее представление для $\widehat{H}$ функции.

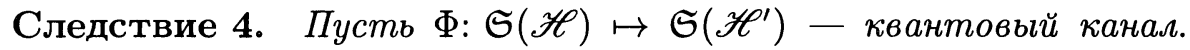
Тогда

$$
\widehat{H}_{\Phi}(\rho)=H_{\Phi}^{* *}(\rho)=\sup _{A \in \mathfrak{B}_{h}(\mathscr{H})} \inf _{\sigma \in \mathfrak{S}(\mathscr{H})}\left(H_{\Phi}(\sigma)+\operatorname{Tr} A(\rho-\sigma)\right)
$$

для любого состояния $\rho \in \mathfrak{S}(\mathscr{H})$.

Рассмотрим множество $\widehat{H}_{\Phi}^{-1}(0)=\left\{\rho \in \mathfrak{S}(\mathscr{H}) \mid \widehat{H}_{\Phi}(\rho)=0\right\}$. Заметим, что множество $H_{\Phi}^{-1}(0)=\left\{\rho \in \mathfrak{S}(\mathscr{H}) \mid H_{\Phi}(\rho)=0\right\}$ является замкнутым подмножеством $\mathfrak{S}(\mathscr{H})$ в силу полунепрерывности снизу квантовой энтропии [34].

Предложение 8. Множество $\widehat{H}_{\Phi}^{-1}(0)$ совпадает с выпукльцм замыканием множества $H_{\Phi}^{-1}(0) \cap \operatorname{Extr} \mathfrak{S}(\mathscr{H})$.

Доказ а т ель с т в о. Пусть $\rho_{0} \in \overline{\mathrm{co}}\left(H_{\Phi}^{-1}(0) \cap \operatorname{Extr} \mathfrak{S}(\mathscr{H})\right)$. Tогда найдется последовательность $\left\{\rho_{n}\right\} \subset \operatorname{co}\left(H_{\Phi}^{-1}(0) \cap \operatorname{Extr} \mathfrak{S}(\mathscr{H})\right)$, сходящаяся к $\rho_{0}$. По определению $\widehat{H}_{\Phi}\left(\rho_{n}\right)=0$. Из неотрицательности и полунепрерывности снизу функции $\widehat{H}_{\Phi}$ (лемма 4) следует $\widehat{H}_{\Phi}\left(\rho_{0}\right)=0$. 
Пусть $\rho_{0} \in \widehat{H}_{\Phi}^{-1}(0)$. В силу предложения 6 состояние $\rho_{0}$ является барицентром некоторой меры $\pi_{0}$ с носителем на множестве чистых состояний и такой, что $H_{\Phi}(\rho)=0$ для $\pi_{0}$-почти всех $\rho$. Используя рассуждения доказательства теоремы 6.3 из [28], легко показать, что меру $\pi_{0}$ можно аппроксимировать последовательностью мер $\left\{\pi_{n}\right\}$ с конечным носителем на множестве чистых состояний такой, что $H_{\Phi}(\rho)=0$ для $\pi_{n}$-почти всех $\rho$. Это значит, что при каждом $n$ все атомы меры $\pi_{n}$ являются чистыми состояниями из $H_{\Phi}^{-1}(0)$. В силу непрерывности отображения $\pi \mapsto \bar{\rho}(\pi)$ состояние $\rho_{0}=\bar{\rho}\left(\pi_{0}\right)$ является пределом последовательности $\left\{\bar{\rho}\left(\pi_{n}\right)\right\}$ состояний из $\operatorname{co}\left(H_{\Phi}^{-1}(0) \cap \operatorname{Extr} \mathfrak{S}(\mathscr{H})\right)$. Предложение 8 доказано.

6. О непрерывности функций $\chi_{\Phi}$ и $\widehat{H}_{\Phi}$. Из леммы 2 следует, что

$$
\chi_{\Phi}(\rho)=H_{\Phi}(\rho)-\widehat{H}_{\Phi}(\rho)
$$

для всех состояний $\rho$ с конечной выходной энтропией. Это выражение остается справедливым, когда $H_{\Phi}(\rho)=+\infty$ и $\widehat{H}_{\Phi}(\rho)<+\infty$. Действительно, подставляя $\widehat{H}_{\Phi}$-оптимальную меру $\pi$ для состояния $\rho$ в выражение (4) из $[10]$, легко получить, что $\chi_{\Phi}(\rho)=+\infty$. Заметим также, что для любого состояния $\rho$ из конечности $\chi_{\Phi}(\rho)$ и $\widehat{H}_{\Phi}(\rho)$ следует конечность $H_{\Phi}(\rho)$, а значит, и справедливость выражения (25). Последнее утверждение нетрудно доказать, используя предложение 4 и лемму 5 .

Из выражения (25) следует, что непрерывность на некотором множестве $\mathscr{A} \subseteq \mathfrak{S}(\mathscr{H})$ функций $\chi_{\Phi}$ и $\widehat{H}_{\Phi}$ гарантирует непрерывность на этом множестве выходной энтропии $H_{\Phi}$. Важный результат состоит в том, что верно и обратное утверждение, которое также следует из выражения (25) с учетом полунепрерывности снизу функции $\chi_{\Phi}$ (предложение 4) и полунепрерывности снизу функции $\widehat{H}_{\Phi}$ (лемма 4 ).

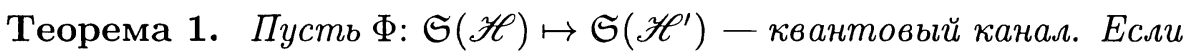
сужение выходной энтропии $H_{\Phi}$ на некоторое множество $\mathscr{A} \subseteq \mathfrak{S}(\mathscr{H})$ непрерьвно, то сужения функиий $\chi_{\Phi} и \widehat{H}_{\Phi}$ на множество $\mathscr{A}$ также непрерьвныл.

3 а м е ч а н и е 3. Отметим определенную парадоксальность утверждения теоремы 1. Дело в том, что значение выходной энтропии $H_{\Phi}$ в некотором состоянии $\rho_{0}$ зависит только от действия канала $\Phi$ на это состояние. Поэтому непрерывность функции $H_{\Phi}$ на некотором множестве $\mathscr{A}$ равносильна непрерывности энтропии на множестве $\Phi(\mathscr{A})$, которая зависит только от вида множества $\Phi(\mathscr{A})$. Напротив, значения функций $\chi_{\Phi}$ и $\widehat{H}_{\Phi}$ в некотором состоянии $\rho_{0}$ зависят, в силу их определения, от действия канала $\Phi$ на все состояния, лежащие в объединении носителей всех мер с барицентром $\rho_{0}$. Поэтому поведение этих функций на некотором множестве $\mathscr{A}$ зависит от действия канала $\Phi$ на все состояния, 
лежащие в объединении носителей всех мер с барицентром в $\mathscr{A}$. Тем не менее, в силу теоремы 1 , непрерывность энтропии на множестве $\Phi(\mathscr{A})$ гарантирует непрерывность функций $\chi_{\Phi}$ и $\widehat{H}_{\Phi}$ на множестве $\mathscr{A}$ независимо от действия канала $\Phi$ на все состояния, не лежащие в $\mathscr{A}$.

Отметим также, что непрерывность каждой из функций $\chi_{\Phi}$ и $\widehat{H}_{\Phi}$ по отдельности на некотором множестве $\mathscr{A}$ не гарантирует непрерывность выходной энтропии на этом множестве. Например, в случае тождественного канала $\Phi$ функция $\widehat{H}_{\Phi}$ тождественно равна нулю на $\mathfrak{S}(\mathscr{H})$, а функция $H_{\Phi}$ - энтропия состояния - разрывна на $\mathfrak{S}(\mathscr{H})$.

Из предложения 3 в [10] вытекает следующее утверждение.

Следствие 5. Пусть $H^{\prime}-$ положительный неограниченный оператор на пространстве $\mathscr{H}^{\prime}$ такой, что $\operatorname{Tr} \exp \left(-\beta H^{\prime}\right)<+\infty$ для всех $\beta>0{ }^{6)}$ Тогда сужения функиий $\chi_{\Phi}$ и $\widehat{H}_{\Phi}$ на подмножество

$$
\mathscr{A}_{h^{\prime}}=\left\{\rho \in \mathfrak{S}(\mathscr{H}) \mid \operatorname{Tr} \Phi(\rho) H^{\prime} \leqslant h^{\prime}\right\}
$$

непрерьвнь при каждом $h^{\prime} \geqslant 0$.

Как отмечалось в [10], условие следствия 5 выполнено для квантовых гауссовских каналов с ограничениями на среднюю энергию вида $\operatorname{Tr} \rho H \leqslant h$, где $H=R^{\mathrm{T}} \varepsilon R$ - гамильтониан системы осцилляторов с невырожденной матрицей энергии $\varepsilon$, а $R$ - канонические переменные системы.

Предложение 9. Пусть $\left\{\rho_{n}\right\}$ - последовательность состояний, сходящаяся $\kappa$ состоянию $\rho_{0}$, такая, что $\lim _{n \rightarrow+\infty} \widehat{H}_{\Phi}\left(\rho_{n}\right)=$ $\widehat{H}_{\Phi}\left(\rho_{0}\right)^{7)}$, a $\pi_{n}-\widehat{H}_{\Phi}$-оптимальная мера для состояния $\rho_{n}$ при каждом $n=1,2 \ldots$ Тогда множество частичных пределов последовательно-

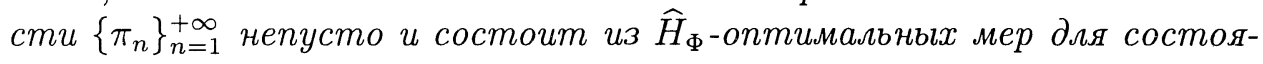
ния $\rho_{0}$.

Д ок а з а т ел ь с т в о. Пусть $\left\{\rho_{n}\right\}$ и $\left\{\pi_{n}\right\}$ - указанные последовательности. Поскольку множество $\left\{\rho_{n}\right\}_{n=0}^{+\infty}$ компактно в $\mathfrak{S}(\mathscr{H})$, множество $\mathscr{P}_{\left\{\left\{\rho_{n}\right\}_{n=0}^{+\infty}\right\}}$ компактно в $\mathscr{P}$ в силу предложения 2 из [10]. Следовательно, последовательность $\left\{\pi_{n}\right\} \subseteq \mathscr{P}_{\left\{\left\{\rho_{n}\right\}_{n=0}^{+\infty}\right\}}$ имеет частичные пределы. Пусть $\pi_{0}$ - предел некоторой подпоследовательности $\left\{\pi_{n_{k}}\right\}$ последовательности $\left\{\pi_{n}\right\}$. В силу полунепрерывности снизу функционала (18) имеем

$$
\begin{aligned}
\widehat{H}_{\Phi}\left(\rho_{0}\right) & =\lim _{k \rightarrow+\infty} \widehat{H}_{\Phi}\left(\rho_{n_{k}}\right)=\lim _{k \rightarrow+\infty} \int_{\mathfrak{S}(\mathscr{H})} H_{\Phi}(\rho) \pi_{n_{k}}(d \rho) \\
& \geqslant \int_{\mathfrak{S}(\mathscr{H})} H_{\Phi}(\rho) \pi_{0}(d \rho)
\end{aligned}
$$

\footnotetext{
6) В [10] такой оператор назван $\mathfrak{H}$-оператором.

7) Например, последовательность состояний конечного ранга, существующая в силу леммы 5 .
} 
что означает $\widehat{H}_{\Phi}$-оптимальность меры $\pi_{0}$ для состояния $\rho_{0}$. Предложение 9 доказано.

Теорема 1 и предложение 9 позволяют представить $\widehat{H}_{\Phi}$-оптимальную ( $\chi_{\Phi}$-оптимальную) меру для любого состояния с конечной выходной энтропией как предельную точку последовательности мер с конечным носителем на множестве чистых состояний, т.е. обычных ансамблей. Напомним, что, в силу предложения 6, для любого состояния конечного ранга $n$ существует $\widehat{H}_{\Phi}$-оптимальная мера с конечным носителем, состоящим не более чем из $n^{2}$ атомов - чистых состояний.

Следствие 6. Пусть $\rho_{0}$ - состояние такое, что $H_{\Phi}\left(\rho_{0}\right)<+\infty$, $P_{n}$ - спектральный проектор $\rho_{0}$, соответствующий $n$ наибольшим собственным значениям, и $\pi_{n}-\widehat{H}_{\Phi}$-оптимальная ( ${ }_{\Phi}$-оптимальная) мера с конечным носителем (ансамбль из $n^{2}$ чистьх состояний) для состояния конечного ранга $\rho_{n}=\left(\operatorname{Tr} P_{n} \rho_{0}\right)^{-1} P_{n} \rho_{0}$ при каждом $n \in \mathbf{N}$. Тогда любой частичный предел последовательности $\left\{\pi_{n}\right\}$ является $\widehat{H}_{\Phi}$-оптимальной $\left(\chi_{\Phi}\right.$-оптимальной) мерой для состояния $\rho_{0}$.

Д о к а з а т е л ь с т в о. Использую теорему Саймона о мажорируемой сходимости [33], легко показать, что

$$
\lim _{n \rightarrow+\infty} H_{\Phi}\left(\rho_{n}\right)=H_{\Phi}\left(\rho_{0}\right)
$$

Следовательно, условия теоремы 1 выполнены для $\mathscr{A}=\left\{\rho_{n}\right\}_{n=0}^{+\infty}$. Поэтому $\lim _{n \rightarrow+\infty} \widehat{H}_{\Phi}\left(\rho_{n}\right)=\widehat{H}_{\Phi}\left(\rho_{0}\right)$, и из предложения 9 получаем утверждение следствия.

7. О проблеме аддитивности. Пусть $\Phi: \mathfrak{S}(\mathscr{H}) \mapsto \mathfrak{S}\left(\mathscr{H}^{\prime}\right)$ и $\Psi: \mathfrak{S}(\mathscr{K}) \mapsto \mathfrak{S}\left(\mathscr{K}^{\prime}\right)$ - каналы с ограничениями, определяемыми множествами $\mathscr{A}$ и $\mathscr{B}$ соответственно. Рассмотрим канал $\Phi \otimes \Psi: \mathfrak{S}(\mathscr{H} \otimes \mathscr{K}) \mapsto$ $\mathfrak{S}\left(\mathscr{H}^{\prime} \otimes \mathscr{K}^{\prime}\right)$. Для этого канала естественно рассмотреть ограничение, определяемое множеством

$$
\mathscr{A} \otimes \mathscr{B}=\left\{\omega \in \mathfrak{S}(\mathscr{H} \otimes \mathscr{K}) \mid \omega^{\mathscr{H}} \in \mathscr{A}, \omega^{\mathscr{K}} \in \mathscr{B}\right\},
$$

где использованы обозначения $\omega^{\mathscr{H}}=\operatorname{Tr}_{\mathscr{K}} \omega$ и $\omega^{\mathscr{K}}=\operatorname{Tr}_{\mathscr{H}} \omega$.

Аддитивность $\chi$-пропускной способности $\mathscr{A}$-ограниченного канала $\Phi$ и $\mathscr{B}$-ограниченного канала $\Psi$ означает равенство

$$
\bar{C}(\Phi \otimes \Psi, \mathscr{A} \otimes \mathscr{B})=\bar{C}(\Phi, \mathscr{A})+\bar{C}(\Psi, \mathscr{B}) .
$$

3 а м е ч а н и е 4 . Пусть $\Omega(\Phi, \mathscr{A})$ и $\Omega(\Psi, \mathscr{B})$ - выходные оптимальные средние состояния для $\mathscr{A}$-ограниченного канала $\Phi$ и $\mathscr{B}$-ограниченного канала $\Psi$ соответственно. Из аддитивности $\chi$-пропускной способности (26) следует, что состояние $\Omega(\Phi, \mathscr{A}) \otimes \Omega(\Psi, \mathscr{B})$ является выходным оптимальным средним для $\mathscr{A} \otimes \mathscr{B}$-ограниченного канала $\Phi \otimes \Psi$. Действительно, пусть $\left\{\left\{\pi_{i}^{k}, \rho_{i}^{k}\right\}\right\}_{k}$ и $\left\{\left\{\mu_{j}^{k}, \sigma_{j}^{k}\right\}\right\}_{k}$ - аппроксимирующие 
последовательности ансамблей для $\mathscr{A}$-ограниченного канала $\Phi$ и $\mathscr{B}$-ограниченного канала $\Psi$. В силу предложения 1 последовательности $\left\{\Phi\left(\bar{\rho}_{k}\right)\right\}_{k}$ и $\left\{\Psi\left(\bar{\sigma}_{k}\right)\right\}_{k}$ сходятся к $\Omega(\Phi, \mathscr{A})$ и $\Omega(\Psi, \mathscr{B})$ соответственно. Из (26) следует, что последовательность ансамблей $\left\{\left\{\pi_{i}^{k} \mu_{j}^{k}, \rho_{i}^{k} \otimes \sigma_{j}^{k}\right\}\right\}_{k}$ является аппроксимирующей для $\mathscr{A} \otimes \mathscr{B}$-ограниченного канала $\Phi \otimes \Psi$. В силу предложения 1 состояние $\Omega(\Phi, \mathscr{A}) \otimes \Omega(\Psi, \mathscr{B})$ - предел последовательности $\left\{\Phi\left(\bar{\rho}_{k}\right) \otimes \Psi\left(\bar{\sigma}_{k}\right)\right\}_{k}$ - является выходным оптимальным средним для $\mathscr{A} \otimes \mathscr{B}$-ограниченного канала $\Phi \otimes \Psi$.

Аддитивность $\chi$-пропускной способности (26) для произвольных множеств-ограничений $\mathscr{A}$ и $\mathscr{B}$ равносильна выполнению следующего неравенства:

$$
\chi_{\Phi \otimes \Psi}(\omega) \leqslant \chi_{\Phi}\left(\omega^{\mathscr{H}}\right)+\chi_{\Psi}\left(\omega^{\mathscr{K}}\right)
$$

для любых состояний $\omega \in \mathfrak{S}(\mathscr{H} \otimes \mathscr{K})$ (субаддитивность $\chi$-функции). Действительно, пусть $\omega-$ произвольное состояние из $\mathfrak{S}(\mathscr{H} \otimes \mathscr{K})$. Положим $\mathscr{A}=\left\{\omega^{\mathscr{H}}\right\}$ и $\mathscr{B}=\left\{\omega^{\mathscr{K}}\right\}$. Тогда из (26) и определения $\chi$-функции следует, что

$$
\begin{aligned}
\chi_{\Phi}\left(\omega^{\mathscr{H}}\right)+\chi_{\Psi}\left(\omega^{\mathscr{K}}\right) & =\bar{C}\left(\Phi,\left\{\omega^{\mathscr{H}}\right\}\right)+\bar{C}\left(\Psi,\left\{\omega^{\mathscr{K}}\right\}\right) \\
& =\bar{C}\left(\Phi \otimes \Psi,\left\{\omega^{\mathscr{H}}\right\} \otimes\left\{\omega^{\mathscr{K}}\right\}\right) \geqslant \chi_{\Phi \otimes \Psi}(\omega) .
\end{aligned}
$$

Обратно, из (27) следует, что

$$
\bar{C}(\Phi \otimes \Psi, \mathscr{A} \otimes \mathscr{B}) \leqslant \bar{C}(\Phi, \mathscr{A})+\bar{C}(\Psi ; \mathscr{B}) .
$$

Поскольку обратное неравенство очевидно, получаем (26). В [31] показано, что субаддитивность $\chi$-функции имеет место для нетривиального класса бесконечномерных каналов.

В работе [13] был предложен подход к проблеме аддитивности в конечномерном случае, основанный на применении техники выпуклого анализа. Результаты предыдущих разделов позволяют распространить этот подход на случай бесконечномерных каналов.

Для канала $\Phi: \mathfrak{S}(\mathscr{H}) \mapsto \mathfrak{S}\left(\mathscr{H}^{\prime}\right)$ и оператора $A \in \mathfrak{B}_{+}(\mathscr{H})$ введем следующую характеристику [30]:

$$
\nu_{H}(\Phi, A)=\inf _{\rho \in \mathfrak{S}(\mathscr{H})}\left(H_{\Phi}(\rho)+\operatorname{Tr} A \rho\right) .
$$

Заметим, что данная характеристика является обобщением минимальной выходной энтропии канала $\Phi$, определяемой выражением

$$
H_{\min }(\Phi)=\inf _{\rho \in \mathfrak{S}(\mathscr{H})} H_{\Phi}(\rho)=\nu_{H}(\Phi, 0)
$$

Из вогнутости квантовой энтропии следует, что точную нижнюю грань в (28) и (29) можно брать по множеству всех чистых состояний $\rho$ из $\mathfrak{S}(\mathscr{H})$. 
Аддитивность минимальной выходной энтропии для каналов $\Phi$ и $\Psi$ означает равенство

$$
H_{\min }(\Phi \otimes \Psi)=H_{\min }(\Phi)+H_{\min }(\Psi),
$$

которое является частным случаем аддитивности введенной выше характеристики канала по отношению к сумме Кронекера:

$$
\nu_{H}(\Phi \otimes \Psi, A \otimes I+I \otimes B)=\nu_{H}(\Phi, A)+\nu_{H}(\Psi, B) .
$$

Если $\Phi$ и $\Psi$ - конечномерные каналы, то, как показано в [21], выполнение неравенства (27) для всех состояний $\omega$ равносильно выполнению неравенства

$$
\widehat{H}_{\Phi \otimes \Psi}(\omega) \geqslant \widehat{H}_{\Phi}\left(\omega^{\mathscr{H}}\right)+\widehat{H}_{\Psi}\left(\omega^{\mathscr{K}}\right)
$$

для всех состояний $\omega$ (супераддитивность $\widehat{H}$-функции). Для бесконечномерного случая соотношения между введенными выше свойствами суб(супер-) аддитивности представлены в следующей теореме.

Пусть $\mathfrak{S}_{\Phi, \Psi}=\left\{\omega \in \mathfrak{S}(\mathscr{H} \otimes \mathscr{K}) \mid H_{\Phi}\left(\omega^{\mathscr{H}}\right)<+\infty, H_{\Psi}\left(\omega^{\mathscr{K}}\right)<+\infty\right\}-$ выпуклое подмножество $\mathfrak{S}(\mathscr{H} \otimes \mathscr{K})$.

Теорема 2. Пусть $\Phi: \mathfrak{S}(\mathscr{H}) \mapsto \mathfrak{S}\left(\mathscr{H}^{\prime}\right) u \Psi: \mathfrak{S}(\mathscr{K}) \mapsto \mathfrak{S}\left(\mathscr{K}^{\prime}\right)-$ произвольные каналь.

1) Выполнение неравенства (32) для всех состояний $\omega \in \mathfrak{S}(\mathscr{H} \otimes \mathscr{K})$ равносильно выполнению равенства (31) для всех положительных операторов $A \in \mathfrak{B}_{+}(\mathscr{H})$ и $B \in \mathfrak{B}_{+}(\mathscr{K})$, из которого следует аддитивность минимальной выходной энтропии (30).

2) Выполнение неравенства (32) для всех состояний $\omega \in \mathfrak{S}_{\Phi, \Psi}$ равносильно выполнению неравенства (27) для всех состояний $\omega \in \mathfrak{S}_{\Phi, \Psi}$, которое означает аддитивность Х-пропускной способности (26) для любых множеств-ограничений $\mathscr{A}$ и $\mathscr{B}$ таких, что

$$
H_{\Phi}(\rho)<+\infty \quad \forall \rho \in \mathscr{A} \quad u \quad H_{\Psi}(\sigma)<+\infty \quad \forall \sigma \in \mathscr{B} .
$$

Д о к а з а т е л ь с т в о. 1) Рассматривая состояния-произведения, легко получить следующее свойство субаддитивности:

$$
\nu_{H}(\Phi \otimes \Psi, A \otimes I+I \otimes B) \leqslant \nu_{H}(\Phi, A)+\nu_{H}(\Psi, B) .
$$

В силу предложения 7 функция $\widehat{H}_{\Phi}$ является выпуклым замыканием функции $H_{\Phi}$. Преобразование Фенхеля $H_{\Phi}^{*}$ функции $H_{\Phi}$ определено на множестве $\mathfrak{B}_{h}(\mathscr{H})$ всех эрмитовых операторов выражением (23). В силу леммы 1 из [13] (формально рассматриваемые функции не удовлетворяют условиям данной леммы, но легко видеть, что все рассуждения в ее доказательстве остаются справедливыми в данном случае), сильная супераддитивность $\widehat{H}$-функции эквивалентна субаддитивности преобразования Фенхеля по отношению к сумме Кронекера:

$$
\begin{gathered}
H_{\Phi \otimes \Psi}^{*}\left(A \otimes I_{\mathscr{K}}+I_{\mathscr{H}} \otimes B\right) \leqslant H_{\Phi}^{*}(A)+H_{\Psi}^{*}(B) \\
\forall A \in \mathfrak{B}_{h}(\mathscr{H}), \quad \forall B \in \mathfrak{B}_{h}(\mathscr{K}) .
\end{gathered}
$$


По определению последнее неравенство равносильно следующему:

$$
\begin{aligned}
& \sup _{\omega \in \mathfrak{S}(\mathscr{H} \otimes \mathscr{K})}\left(\operatorname{Tr} A \omega^{\mathscr{H}}+\operatorname{Tr} B \omega^{\mathscr{K}}-H(\Phi \otimes \Psi(\omega))\right) \\
& \quad \leqslant \sup _{\rho \in \mathfrak{S}(\mathscr{H})}(\operatorname{Tr} A \rho-H(\Phi(\rho)))+\sup _{\sigma \in \mathfrak{S}(\mathscr{K})}(\operatorname{Tr} B \sigma-H(\Psi(\sigma)))
\end{aligned}
$$

для всех $A \in \mathfrak{B}_{h}(\mathscr{H})$ и $B \in \mathfrak{B}_{h}(\mathscr{K})$.

Учитывая инвариантность предыдущего неравенства относительно замены $A$ и $B$ на $A \pm\|A\| I_{\mathscr{H}}$ и $B \pm\|B\| I_{\mathscr{K}}$ соответственно и используя (33), получаем утверждение 1 ) теоремы.

2) В силу представления (25) и субаддитивности квантовой энтропии, из неравенства (32) для любого состояния $\omega \in \mathfrak{S}_{\Phi, \Psi}$ следует неравенство (27) для этого состояния.

Предположим, что неравенство (27) имеет место для любого состояния $\omega \in \mathfrak{S}_{\Phi, \Psi}$. В силу наблюдения, приведенного после замечания 4 , это означает аддитивность $\chi$-пропускной способности (26) для любых $\mathscr{A}$ и $\mathscr{B}$ таких, что $H_{\Phi}(\rho)<+\infty$ для всех $\rho \in \mathscr{A}$ и $H_{\Psi}(\sigma)<+\infty$ для всех $\sigma \in \mathscr{B}$. В частности, $\bar{C}\left(\Phi \otimes \Psi,\left\{\omega^{\mathscr{H}}\right\} \otimes\left\{\omega^{\mathscr{K}}\right\}\right)=\bar{C}\left(\Phi,\left\{\omega^{\mathscr{H}}\right\}\right)+$ $\bar{C}\left(\Psi,\left\{\omega^{\mathscr{K}}\right\}\right)$.

В силу замечания 3 состояние $\Phi\left(\omega^{\mathscr{H}}\right) \otimes \Psi\left(\omega^{\mathscr{K}}\right)$ является выходным оптимальным средним для $\left\{\omega^{\mathscr{H}}\right\} \otimes\left\{\omega^{\mathscr{K}}\right\}$-ограниченного канала $\Phi \otimes \Psi$. Учитывая, что $\omega \in\left\{\omega^{\mathscr{H}}\right\} \otimes\left\{\omega^{\mathscr{K}}\right\}$, и используя предложение 3 , получаем

$$
\begin{aligned}
\chi_{\Phi}\left(\omega^{\mathscr{H}}\right)+\chi_{\Psi}\left(\omega^{\mathscr{K}}\right) & =\bar{C}\left(\Phi,\left\{\omega^{\mathscr{H}}\right\}\right)+\bar{C}\left(\Psi,\left\{\omega^{\mathscr{K}}\right\}\right) \\
& =\bar{C}\left(\Phi \otimes \Psi,\left\{\omega^{\mathscr{H}}\right\} \otimes\left\{\omega^{\mathscr{K}}\right\}\right) \\
& \geqslant \chi_{\Phi \otimes \Psi}(\omega)+H\left((\Phi \otimes \Psi)(\omega) \| \Phi\left(\omega^{\mathscr{H}}\right) \otimes \Psi\left(\omega^{\mathscr{K}}\right)\right) .
\end{aligned}
$$

Поскольку

$$
\begin{aligned}
& H\left((\Phi \otimes \Psi)(\omega) \| \Phi\left(\omega^{\mathscr{H}}\right) \otimes \Psi\left(\omega^{\mathscr{K}}\right)\right) \\
& \quad=H\left(\Phi\left(\omega^{\mathscr{H}}\right)\right)+H\left(\Psi\left(\omega^{\mathscr{K}}\right)\right)-H((\Phi \otimes \Psi)(\omega)),
\end{aligned}
$$

неравенство (34) вместе с (25) приводит к (32). Теорема 2 доказана.

В отличие от конечномерного случая теорема 2 не позволяет показать, что из субаддитивности $\chi$-функции (выполнение неравенства (27) для всех состояний $\omega$ ) следует супераддитивность $\hat{H}$-функции (выполнение неравенства (32) для всех состояний $\omega$ ) для двух произвольных каналов и наоборот. Однако для нетривиального класса каналов с конечной выходной энтропией (см. пример в [3]) из этой теоремы следует эквивалентность данных свойств.

Следствие 7. Для любых двух каналов $\Phi$ и $\Psi$ с конечной выходной энтропией субаддитивность ұ-Функиии равносильна супераддитивности $\widehat{H}$-функиии. 
Основная трудность, не позволяющая доказать аналогичное утверждение для произвольных каналов, связана с существованием «суперсцепленных» чистых состояний $\omega$, имеющих частичные следы с бесконечной энтропией (см. замечание 4 в [31]).

8. Об определении меры сцепленности. Сцепленность - специфическая черта составных квантовых систем. Одной из количественных характеристик сцепленности является сцепленность формирования $(\mathrm{EoF})[17]$. В конечномерном случае $\mathrm{EoF}$ определяется следующим образом:

$$
E_{F}(\rho)=\min _{\sum_{i} \pi_{i} \rho_{i}=\rho} \sum_{i} \pi_{i} H_{\Phi}\left(\rho_{i}\right),
$$

где $\Phi$ - частичный след - канал из пространства состояний составной системы на пространство состояний подсистемы. В терминах выпуклого анализа EoF совпадает с выпуклой оболочкой выходной энтропии частичного следа. Непрерывность ЕoF, установленная в [26], показывает, что эта выпуклая оболочка является замкнутой, т.е. ЕоF есть выпуклое замыкание выходной энтропии частичного следа.

Обобщение этого понятия на бесконечномерный случай было рассмотрено в [19], где ЕoF определяется выражением

$$
E_{F}^{D}(\rho)=\inf _{\sum_{i} \pi_{i} \rho_{i}=\rho} \sum_{i} \pi_{i} H_{\Phi}\left(\rho_{i}\right),
$$

в котором точная нижняя грань берется по всем счетным ансамблям чистых состояний со средним состоянием $\rho$.

Альтернативный подход к определению EoF предложен в [24] в случае тензорного произведения двух гильбертовых пространств, одно из которых является конечномерным. Полученные в предыдущих разделах результаты позволяют развить этот подход и определить ЕоF в общем случае следующим образом:

$$
E_{F}^{C}(\rho)=\widehat{H}_{\Phi}(\rho)=\inf _{\pi \in \mathscr{P}_{\{\rho\}}} \int_{\mathfrak{S}(\mathscr{H})} H_{\Phi}(\rho) \pi(d \rho),
$$

где $\Phi$ - частичный след.

Предложение 7 показывает, что $E_{F}^{C}$ - выпуклая полунепрерывная снизу функция, совпадающая с выпуклым замыканием выходной энтропии частичного следа. Из предложения 6 следует, что точная нижняя грань в приведенном выше выражении для EoF достигается на некоторой мере с носителем на множестве чистых состояний. Предложение 8 гарантирует следующее естественное свойство меры сцепленности $E_{F}^{C}$ :

$$
\left\{E_{F}^{C}(\rho)=0\right\} \Longleftrightarrow\{\text { состояние } \rho \text { разделимо }\},
$$

где множество разделимых (несцепленных) состояний определяется как выпуклое замыкание чистых состояний-произведений [3]. Действительно, если $\Phi$ - частичный след, то множество $H_{\Phi}^{-1}(0) \cap \operatorname{Extr} \mathfrak{S}(\mathscr{H})$ совпадает с множеством всех чистых состояний-произведений. Теорема 1 
гарантирует непрерывность $E_{F}^{C}$ на подмножествах состояний, на которых непрерывна выходная энтропия одного из частичных следов. В силу предложения 3 из [19] таким свойством обладает важное для физических приложений множество всех состояний с ограниченной средней энергией. Отметим также, что из теоремы 1 следует непрерывность $E_{F}^{C}=E_{F}^{D}$ на всем пространстве состояний составной системы, одна из подсистем которой конечномерна. (Доказательство непрерывности ЕоF нетривиально даже в конечномерном случае [26].)

Интересный вопрос - это соотношение между $E_{F}^{D}$ и $E_{F}^{C}$. Из предложения 6 следует, что

$$
E_{F}^{D}(\rho) \geqslant E_{F}^{C}(\rho)
$$

для всех состояний $\rho$. Поскольку произвольное состояние можно представить как счетную выпуклую комбинацию чистых состояний, лемма 2 и вогнутость выходной энтропии гарантируют равенство

$$
E_{F}^{D}(\rho)=E_{F}^{C}(\rho)
$$

для всех состояний $\rho$, у которых один из частичных следов имеет конечную энтропию. Легко видеть, что (35) имеет место для всех несцепленных и всех чистых состояний (для которых $\widehat{H}_{\Phi}$ совпадает с $H_{\Phi}$ ). Заметим, что из леммы 5 следует, что

$$
E_{F}^{C}(\rho)=\lim _{\varepsilon \rightarrow+0} \inf _{\sum_{i} \pi_{i} \rho_{i} \in \mathscr{U}_{\varepsilon}(\rho)} \sum_{i} \pi_{i} H_{\Phi}\left(\rho_{i}\right),
$$

где $\mathscr{U}_{\varepsilon}(\rho)-\varepsilon$-окрестность состояния $\rho$ и точная нижняя грань берется по всем конечным ансамблям чистых состояний. Однако справедливость равенства (35) для смешанных состояний, у которой оба частичных следа имеют бесконечную энтропию, остается открытой проблемой. В приложении Б показано, что справедливость этого равенства нельзя доказать, используя только общие функциональные свойства выходной энтропии.

\section{9. Приложения}

А. Выпуклая оболочка и выпуклое замыкание. В этом разделе собраны необходимые понятия и факты из выпуклого анализа, в изложении которых мы будем следовать [4]. Пусть $f$ - произвольная вещественная функция на замкнутом выпуклом подмножестве $X$ некоторого локально выпуклого линейного топологического пространства. Рассмотрим надграфик ері $(f)=\{(x, \lambda) \in X \times \mathbf{R} \mid \lambda \geqslant f(x)\} \subseteq X \times \mathbf{R}$. 3аметим, что функция $f$ однозначно определяется своим надграфиком ері $(f)$. Функция $f$ называется выпуклой, если ее надграфик ері $(f)$ является выпуклым подмножеством $X \times \mathbf{R}$. Функция $f$ называется замкнутой, если 
ее надграфик ері $(f)$ является замкнутым подмножеством $X \times \mathbf{R}$. Функция $f$ называется собственной, если она не принимает значения $-\infty$. Для собственной функции $f$ выпуклость означает

$$
f\left(\lambda x_{1}+(1-\lambda) x_{2}\right) \leqslant \lambda f\left(x_{1}\right)+(1-\lambda) f\left(x_{2}\right) \quad \forall x_{1}, x_{2} \in X, \quad \forall \lambda \in[0,1] .
$$

Замкнутость функции $f$ равносильна ее полунепрерывности снизу, которая означает замкнутость в $X$ подмножеств, определяемых неравенством $f(x) \leqslant \lambda$ при любых $\lambda \in \mathbf{R}$, что равносильно неравенству

$$
\liminf _{n \rightarrow+\infty} f\left(x_{n}\right) \geqslant f\left(x_{0}\right)
$$

для любой последовательности $\left\{x_{n}\right\}$ точек из $X$, сходящейся к некоторой точке $x_{0}$.

Пусть $f$ - произвольная функция на $X$. Выпуклая оболочка co $f$ функции $f$ определяется выражением

$$
\operatorname{co} f(x)=\inf _{(x, \lambda) \in \operatorname{co}(\operatorname{epi}(f))} \lambda
$$

в котором символ со в правой части обозначает выпуклую оболочку множества. Это эквивалентно следующему представлению:

$$
\operatorname{co} f(x)=\inf _{\sum_{i} \pi_{i} x_{i}=x} \sum_{i} \pi_{i} f\left(x_{i}\right), \quad \pi_{i}>0, \quad \sum_{i} \pi_{i}=1 .
$$

Таким образом, со $f$ - наибольшая выпуклая функция, мажорируемая функцией $f$. Выпуклое замыкание $\overline{\text { со }} f$ функции $f$ определяется соотношением ері $(\overline{\mathrm{co}} f)=\overline{\mathrm{co}}(\operatorname{epi}(f))$, в котором символ $\overline{\mathrm{co}}$ в правой части обозначает замыкание выпуклой оболочки множества. Следовательно, $\overline{\mathrm{co}} f$ - наибольшая выпуклая и замкнутая функция, мажорируемая функцией $f$. Из вышесказанного вытекает следующее неравенство:

$$
\overline{\operatorname{co}} f(x) \leqslant \operatorname{co} f(x) \leqslant f(x) \quad \forall x \in X .
$$

Если $f$ - непрерывная функция на компактном выпуклом множестве $X$ то $\overline{\operatorname{co}} f=\operatorname{cof}[12]$.

Для произвольной вещественной функции $f$ на локально-выпуклом вещественном линейном топологическом пространстве $X$ преобразование Фенхеля $f^{*}$ - это функция на двойственном пространстве $X^{*}$, определяемая выражением

$$
f^{*}(y)=\sup _{x \in X}(\langle y, x\rangle-f(x)) \quad \forall y \in X^{*} .
$$

Двойное преобразование Фенхеля $f^{* *}$ — это функция на пространстве $X$, определяемая выражением

$$
f^{* *}(x)=\sup _{y \in X^{*}}\left(\langle y, x\rangle-f^{*}(y)\right) \quad \forall x \in X .
$$

В силу теоремы Фенхеля $f^{* *}(x)=\overline{\mathrm{co}} f$ для любой собственной функции $f$. Следовательно, для собственной функции $f$ ее выпуклое замыкание $\overline{\text { со }} f$ совпадает с верхней гранью всех непрерывных аффинных функций, мажорируемых функцией $f$. 
Б. О совпадении двух определений ЕoF. Многие вопросы, связанные с понятием квантовой энтропии $H(\rho)$, удается решить, используя следующие свойства этой величины как функции состояния:

- неотрицательность;

- вогнутость;

- полунепрерывность снизу.

Все перечисленные свойства вытекают из более глубокого свойства квантовой энтропии: функция $H(\rho)$ является поточечным пределом возрастающей последовательности неотрицательных вогнутых непрерывных и ограниченных ${ }^{8)}$ функций $H\left(P_{n} \rho P_{n}\right)+\operatorname{Tr} \rho P_{n} \log \operatorname{Tr} \rho P_{n}$, где $P_{n}$ возрастающая последовательность проекторов конечного ранга, сильно сходящаяся к тождественному оператору [22].

Выходная энтропия $H_{\Phi}(\rho)$ любого квантового канала $\Phi$, в частности, частичного следа также обладает всеми указанными выше свойствами, которые позволили получить многие приведенные в данной статье результаты. Например, представление функции $H_{\Phi}(\rho)$ как предела монотонной последовательности вогнутых непрерывных функций позволило показать существование $\widehat{H}_{\Phi}$-оптимальной меры с носителем на множестве чистых состояний (предложение 6).

Цель данного раздела - показать, что указанных выше свойств выходной энтропии $H_{\Phi}(\rho)$ недостаточно для доказательства равенства (35) для всех состояний.

Пусть $\mathscr{E}$ - множество всех функций на $\mathfrak{S}(\mathscr{H})$, представимых в виде поточечного предела монотонно возрастающей последовательности неотрицательных вогнутых непрерывных и ограниченных функций на $\mathfrak{S}(\mathscr{H})$.

Из данного определения следует, что все функции класса $\mathscr{E}$ являются вогнутыми полунепрерывными снизу функциями на $\mathfrak{S}(\mathscr{H})$ со значениями в $[0,+\infty]$.

Предложение. $B$ классе $\mathscr{E}$ существует ограниченная функция $F$ такая, что

$$
\inf _{\sum_{i} \pi_{i} \rho_{i}=\rho_{0}} \sum_{i} \pi_{i} F\left(\rho_{i}\right)=1 \quad u \quad \inf _{\pi \in \mathscr{P}_{\left\{\rho_{0}\right\}}} \int_{\mathfrak{S}(\mathscr{H})} F(\rho) \pi(d \rho)=0
$$

для некоторого состояния $\rho_{0}$ из $\mathfrak{S}(\mathscr{H})$, где точная нижняя грань в первом выражении берется по всем счетным разложениям состояния $\rho_{0}$.

В доказательстве этого предложения сушественную роль играет следующая лемма, в которой вводится индикаторная функция произвольного множества чистых состояний.

8) Ввиду некомпактности $\mathfrak{S}(\mathscr{H})$ из непрерывности функции на $\mathfrak{S}(\mathscr{H})$ не следует ее ограниченность. 
Лемма. Пусть $\mathscr{A}-$ произвольное множество чистых состояний из $\mathfrak{S}(\mathscr{H})$. Функиия $f_{\mathscr{A}}(\rho)=\inf _{\sigma \in \mathscr{A}}(1-\operatorname{Tr} \rho \sigma)$ непрерьвна и вогнута на $\mathfrak{S}(\mathscr{H})$, причем $0 \leqslant f(\rho) \leqslant 1$ u $f^{-1}(0)=\overline{\mathscr{A}}$.

Д о к а з а т е л ь с т в о. Достаточно установить выпуклость и непрерывность функции $1-f(\rho)=g(\rho)=\sup _{\sigma \in \mathscr{A}} \operatorname{Tr} \rho \sigma$, поскольку остальные свойства легко проверяются. Выпуклость и полунепрерывность снизу функции $g(\rho)$ следуют из ее представления как верхней грани семейства $\{\operatorname{Tr} \rho \sigma\}_{\sigma \in \mathscr{A}}$ непрерывных аффинных функций на $\mathfrak{S}(\mathscr{H})$.

Предположим, что функция $g(\rho)$ не является полунепрерывной сверху. Это означает существование последовательности состояний $\left\{\rho_{n}\right\}$, сходящейся к некоторому состоянию $\rho_{0}$, такой, что

$$
\lim _{n \rightarrow+\infty} g\left(\rho_{n}\right)>g\left(\rho_{0}\right) \text {. }
$$

Пусть $\mathfrak{A}=\{|\varphi\rangle \in \mathscr{H}|| \varphi\rangle\langle\varphi| \in \mathscr{A}\}-$ множество единичных векторов из $\mathscr{H}$, а $\overline{\mathfrak{A}}$ - замыкание этого множества в слабой топологии гильбертова пространства $\mathscr{H}$. В силу леммы 2 из $[5, \text { с. } 284]^{9)}$ имеем

$$
g\left(\rho_{0}\right)=\sup _{\sigma \in \mathscr{A}} \operatorname{Tr} \rho_{0} \sigma=\sup _{\varphi \in \mathfrak{A}}\left\langle\varphi\left|\rho_{0}\right| \varphi\right\rangle=\sup _{\varphi \in \overline{\mathfrak{A}}}\left\langle\varphi\left|\rho_{0}\right| \varphi\right\rangle .
$$

Для любого $\varepsilon>0$ и любого натурального $n$ существует вектор $\varphi_{n}^{\varepsilon}$ из $\mathfrak{A}$ такой, что $\left\langle\varphi_{n}^{\varepsilon}\left|\rho_{n}\right| \varphi_{n}^{\varepsilon}\right\rangle>g\left(\rho_{n}\right)-\varepsilon$. Поскольку единичный шар пространства $\mathscr{H}$ компактен в слабой топологии, существует подпоследовательность $\left\{\varphi_{n_{k}}^{\varepsilon}\right\}_{k}$ последовательности $\left\{\varphi_{n}^{\varepsilon}\right\}_{n}$, слабо сходящаяся к некоторому вектору $\varphi_{0}^{\varepsilon} \in \overline{\mathfrak{A}}$. В силу упомянутой выше леммы 2 из [5] последовательность $\left\{\left\langle\varphi_{n_{k}}^{\varepsilon}\left|\rho_{0}\right| \varphi_{n_{k}}^{\varepsilon}\right\rangle\right\}_{k}$ сходится к $\left\langle\varphi_{0}^{\varepsilon}\left|\rho_{0}\right| \varphi_{0}^{\varepsilon}\right\rangle$ при $k \rightarrow+\infty$. Поэтому, с учетом оценки $\left|\left\langle\varphi_{n_{k}}^{\varepsilon}\left|\rho_{n_{k}}-\rho_{0}\right| \varphi_{n_{k}}^{\varepsilon}\right\rangle\right| \leqslant\left\|\rho_{n_{k}}-\rho_{0}\right\|_{1}$, получаем

$$
\lim _{k \rightarrow+\infty} g\left(\rho_{n_{k}}\right) \leqslant \lim _{k \rightarrow+\infty}\left\langle\varphi_{n_{k}}^{\varepsilon}\left|\rho_{n_{k}}\right| \varphi_{n_{k}}^{\varepsilon}\right\rangle-\varepsilon=\left\langle\varphi_{0}^{\varepsilon}\left|\rho_{0}\right| \varphi_{0}^{\varepsilon}\right\rangle-\varepsilon \leqslant g\left(\rho_{0}\right)-\varepsilon,
$$

где последнее неравенство следует из (37). В силу произвольности выбора $\varepsilon$ данное неравенство противоречит предположению (36). Лемма доказана.

Д ок а затель с т в о п ред ло ж ен и я. Пусть $\mathscr{A}_{s}-$ множество всех чистых состояний-произведений в тензорном произведении двух сепарабельных гильбертовых пространств, а $\rho_{0}$ - несцепленное состояние, построенное в [3], такое, что любая мера с барицентром $\rho_{0}$ не может иметь атомов в $\mathscr{A}_{s}$. Пусть $F$ - характеристическая функция дополнения множества $\mathscr{A}_{s}$. Заметим, что при каждом $n=1,2, \ldots$ функция $F_{n}(\rho)=\sqrt[n]{f_{\mathscr{A}_{s}}(\rho)}$, где $f_{\mathscr{A}_{s}}(\rho)$ - функция из приведенной выше леммы, непрерывна и вогнута. Следовательно, поточечный предел $F$ последовательности $\left\{F_{n}\right\}$ является функцией из $\mathscr{E}$. В силу леммы 1 из [3]

9) Данная лемма утверждает, что для произвольной последовательности векторов $\left\{x_{n}\right\}$ в гильбертовом пространстве, слабо сходящейся к вектору $x_{0}$, и для произвольного компактного оператора $A$ существует $\lim _{n \rightarrow+\infty}\left\langle x_{n}|A| x_{n}\right\rangle=\left\langle x_{0}|A| x_{0}\right\rangle$. 
существует (чисто неатомическая) мера $\pi_{0}$ с носителем в $\mathscr{A}_{s}$, имеющая

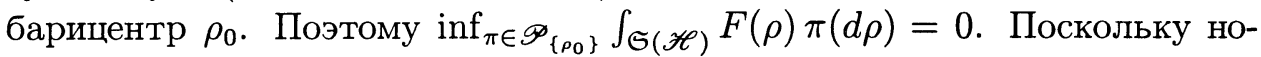
ситель любой атомической меры с барицентром $\rho_{0}$ не пересекается с $\mathscr{A}_{s}$, нетрудно видеть, что inf $\sum_{i_{i} \pi_{i} \rho_{i}=\rho_{0}} \sum_{i} \pi_{i} F\left(\rho_{i}\right)=1$.

Автор благодарен А. С. Холево за постоянную помощь в процессе работы над данной статьей. Автор также благодарен М. Вольфу за полезные замечания.

\section{СПИСОК ЛИТЕРАТУРЫ}

1. Биллингсли П. Сходимость вероятностных мер. М.: Мир, 1977.

2. Браттели У., Робинсон Д. Операторные алгебры и квантовая статистическая механика. М.: Мир, 1982, 511 с.

3. Вернер Р. Ф., Холево А.С., Широков М. Е. О понятии сцепленности в гильбертовых пространствах. - Успехи матем. наук, 2005, т. 60, № 2, с. 153-154.

4. Иоффе А.Д., Тихомиров В.М. Теория экстремальных задач. М.: Наука, 1974, $479 \mathrm{c}$.

5. Колмогоров A.H., Фомин С.В. Элементы теории функций и функционального анализа. М.: Наука, 1989, 623 с.

6. Половинкин Е. С., Балашов М. В. Элементы выпуклого и сильно выпуклого анализа. М.: Физматлит, 2004, 415 с.

7. Холево А.С. Введение в квантовую теорию информации. М.: МЦНМО, 2002, $126 \mathrm{c}$.

8. Холево А.С. Статистическая структура квантовой теории. М.-Ижевск: Ин-т компьют. исслед., 2003, 191 с.

9. Холево А. С. Классическая пропускная способность квантовых каналов с ограничениями. - Теория вероятн. и ее примен., 2003, т. 48, в. 2, с. 359-374.

10. Холево A.C., Широков М. Е. Непрерывные ансамбли и классическая пропускная способность квантовых каналов бесконечной размерности. - Теория вероятн. и ее примен., 2005 , т. 50 , в. 1 , с. $98-114$.

11. Широков M. Е. Энтропийные характеристики подмножеств состояний, I. - Изв. PAH, 2006, т. 70, № 6, c. 193-222.

12. Alfsen E. Compact Convex Sets and Boundary Integrals. New York-Heidelberg: Springer-Verlag, $1971,210 \mathrm{p}$.

13. Audenaert K. M. R., Braunstein S. L. On strong subadditivity of the entanglement of formation. - Comm. Math. Phys., 2004, v. 246, № 3, p. 443-452.

14. Bourgin R.D. Partial orderings for integral representations on convex sets with the Radon-Nikodým property. - Pacific J. Math., 1979, v. 81, № 1, p. 29-44.

15. Donald M. J. Further results on the relative entropy. - Math. Proc. Cambridge Philos. Soc., 1987, v. 101, № 2, p. 363-373.

16. Dell'Antonio G. F. On the limits of sequences of normal states. - Comm. Pure Appl. Math., 1967, v. 20, p. 413-429.

17. Bennett C.H., DiVincenzo D.P., Smolin J.A., Wootters W.K. Mixed-state entanglement and quantum error correction. - Phys. Rev. A (3), 1996, v. 54, № 5, p. $3824-3851$.

18. Davies E. B. Information and quantum measurement. - IEEE Trans. Inform. Theory, 1978 , v. 24 , № 5 , p. $596-599$.

19. Eisert J., Simon C., Plenio M. B. On the quantification of entanglement in infinitedimensional quantum systems. - J. Phys. A, 2002, v. 35, № 17, p. 3911-3923.

20. Harremoës $P$. Information topologies with applications. - Entropy, Search, Complexity. Ed. by I. Csiszár, G. O. H. Katona, and G. Tardos. Berlin: SpringerVerlag, 2007, p. 113-150. (Bolyai Soc. Math. Stud., v. 16.)

21. Holevo A.S., Shirokov M. E. On Shor's channel extension and constrained channels. Comm. Math. Phys., 2004, v. 249, № 2, p. 417-430. 
22. Lindblad $G$. Expectation and entropy inequalities for finite quantum systems. Comm. Math. Phys., 1974, v. 39, №2, p. 111-119.

23. Lindblad G. Completely positive maps and entropy inequalities. - Comm. Math. Phys., 1975, v. 40, № 2, p. 147-151.

24. Majewski A.W. On entanglement of formation. - J. Phys. A, 2002, v. 35, № 1, p. $123-134$.

25. Nielsen M.A., Chuang I.L. Quantum Computation and Quantum Information. Cambridge: Cambridge Univ. Press, 2000, 676 p.

26. Nielsen M.A. Continuity bounds for entanglement. - http://arxiv.org: quant$\mathrm{ph} / 9908086,1999$.

27. Ohya M., Petz D. Quantum Entropy and Its Use. Berlin: Springer-Verlag, 1993, 335 p.

28. Parthasarathy K. R. Probability Measures on Metric Spaces. New York-London: Academic Press, 1967, 276 p.

29. Schumacher B., Westmoreland M. D. Optimal signal ensembles. - http://arxiv.org: quant-ph/9912122, 1999.

30. Shirokov M. E. On the additivity conjecture for channels with arbitrary constraints. http://arxiv.org: quant-ph/0308168, 2003.

31. Shirokov $M$. E. The Holevo capacity of infinite dimensional channels and the additivity problem. - Comm. Math. Phys., 2006, v. 262, № 1, p. 137-159.

32. Shirokov M. E. On channels with finite Holevo capacity. - http://arxiv.org: quant$\mathrm{ph} / 0602073,2006$.

33. Simon B. Convergence theorem for entropy. Appendix in Lieb E. H., Ruskai M. B. Proof of the strong subadditivity of quantum-mechanical entropy. - J. Math.Phys., 1973, v. 14, p. 1938-1941.

34. Wehrl A. General properties of entropy. - Rev. Modern Phys., 1978, v. 50, № 2, p. 221-260.

35. Uhlmann A. Entropy and optimal decompositions of states relative to a maximal commutative subalgebra. - http://arxiv.org: quant-ph/9704017, 1997.

Поступила в редакцию

4.IX.2005 